\title{
Two-noded zigzag beam element accounting for shear effects based on an extended Euler Bernoulli theory
}

\author{
Daniel Di Capua ${ }^{1,3}$ and Eugenio Oñate ${ }^{2,3}$ \\ ${ }^{1}$ Escola Universitària d'Enginyeria Tècnica Industrial (EUETIB), Universidad Politécnica de Cataluña (UPC), Comte d’Urgell \\ 187, 08036 Barcelona, Spain \\ ${ }^{2}$ ETS Ingenieros de Caminos, Canales y Puertos, Universidad Politécnica de Cataluña (UPC), Edificio C1, Campus Norte, UPC, \\ Jordi Girona 1-3, 08034 Barcelona, Spain \\ ${ }^{3}$ International Center for Numerical Methods in Engineering (CIMNE), Gran Capitán s/n, 08034 Barcelona, Spain
}

daniel.di.capua@upc.edu ,onate@cimne.upc.edu

June 4, 2015

\begin{abstract}
We present a new 2-noded beam element based on the refined zigzag theory and the classical EulerBernoulli beam theory for the static analysis of composite laminate and sandwich beams. The proposed element is able to take into account distortion effects due to shear elastic strains and can predict delamination. The element has four degrees of freedom per node. A $C^{1}$ cubic Hermite interpolation is used for the vertical deflection while a $C^{0}$ linear interpolation is employed for the other kinematics variables. The stiffness matrix and the load vector are calculated in explicit form using exact integration. The element is free from shear locking as confirmed with numerical tests on a wide range of the slenderness ratios. Numerical results show the ability of the EEBZ2 element to reproduce accurately the vertical deflection along the beam length and complex zigzag distributions of the axial displacement and the stresses across the thickness. Delamination effects are modeled by incorporating of an additional zigzag function corresponding to the kinematics of a zero thickness layer where delamination occurs. An example showing the capability of the new EEBZ2 element for accurately reproducing delamination effects is presented.
\end{abstract}

Version 2.0: January 2015

Keywords: Refined zigzag theory, extended Euler Bernoulli beam theory, EEBZ2 element, beam finite element, composite beam, sandwich beam.

\section{1-) Introduction}

In the structural analysis of homogeneous linear elements the classical beam theories of EulerBernoulli and Timoshenko [1] are typically used. Both of these theories are based on the assumption that the cross plane sections before deformation are kept plane after deformation. 
This hypothesis leads to a linear variation of the axial displacement field. However it is well know that this assumption does not hold for composite laminate or sandwich beams that have layers with very different mechanical properties. In this case complex variations of the axial displacement field can be found.

The most accurate numerical approach to solve this problem is a 3D finite element formulation. However, the computational cost of this approach is prohibitive in the case of a large number of layers under dynamics or nonlinear situations.

An interesting option to avoid 3D modeling is incorporating high-order terms with respect to the thickness coordinate in the beam formulation. This leads to the so-called equivalent single layer (SL) theories [2]. However despite being computationally efficient, SL theories in some cases generate inaccurate distributions of the strains and stresses through the thickness.

Another popular option is using layer-wise theories [2] in which the distribution of the displacements is defined layer by layer. Layer-wise theories accurately predict the thickness distribution of stresses and strains. However, the number of kinematic variables is proportional to the number of layers which increases the computational cost significantly [3-5].

The so-called zigzag theories are a subgroup of the general layer-wise theory in which the number of kinematic variables is independent of the number of layers. These theories assume that the axial displacement is the superposition of a linear piecewise zigzag distribution and a linear [6,7], quadratic [8,9] or cubic [10-13] in-plane displacement field. In the most of early zigzag theories the zigzag function is obtained by enforcing the continuity of the transverse shear stresses across the laminate thickness.

Zigzag theories typically have as baseline the kinematics assumptions of the classical EulerBernoulli [14,15] and Timoshenko [16-19] beam theories (EBT and TBT). Some researchers consider that the $C^{1}$ continuity requirement for the deflection field of the EBT is a drawback versus the simpler $C^{0}$ requirement of the TBT $[18,20]$. The main reason for this consideration is the theoretical difficulty for the $C^{1}$ approximation to satisfy the equilibrium of forces at clamped supports.

Tessler et al.[18,19] have recently developed a Refined Zigzag Theory (RZT) for beams and plates based in the TBT kinematic assumptions. In this theory the zigzag functions have the property of vanishing on the top and bottom surfaces of the laminate. An additional characteristic of this theory is that the transverse shear stresses are not required to be continuous across the layer interfaces. Then the shear stress distribution is defined by simple piecewise-constant functions that approximate the true distribution in an average sense. A more accurate thickness distribution of the shear stress can be obtained via a post-processing of the axial stress field by integrating the equilibrium equations [20].

Based in the RZT different two and three-noded $C^{0}$ beam elements have been developed [20-23] for the analysis of multilayered composites and sandwich beams.

The zigzag theories have been also used to model delamination in beams and plates. A C0 plate element for delamination analysis based on a zigzag model has been developed by Icardi and Zardo [24]. Cho and Kim [25,26] developed a unified approach to modeling the two-dimensional imperfections and delaminations present in laminated composite plates and shells. Oh et al. [27] have proposed a model for the analysis of the dynamic response of delaminated composite plates based on a higher-order zigzag theory. Other approaches based on higher-order zig-zag approximations for modeling interlayer slips resulting from the delamination related damage have been explored in [28-33]. 
Several others class of beam models have been proposed in the last decades. Some of the most relevant works are the Variational Asymptotic Beam Section Analysis (VABS) [34], the Generalized Beam theories (GBT) [35] and the Carrera Unified Formulation (CUF) [36].

In this paper we present a two-noded beam element based in the RZT and the EBT (here termed EEBZ2) for the analysis of composites laminate and sandwich beams. The proposed model takes into account distortion effects due to the shear strains and can also predict delamination. We analyze the locking-free behavior of the EEBZ2 element for a wide range of the beam slenderness ratios. Numerical examples of simply supported and clamped beams with different laminate patterns are presented to show the excellent performance of the EEBZ2 element. Finally, an example showing the capability of the proposed element to model delamination is presented.

\section{2-) Refined zigzag theory for the classical EBT}

\section{2-1) Displacement field}

Consider a composite laminated beam of length $L$, width $b$ and thickness $h$, made of $n_{\text {layers }}$ orthotropic layers. The beam segment is defined in the $(x, z)$ Cartesian system, where $x$ is the beam longitudinal axis and $z$ is the thickness coordinate. The superscript $k$ denotes quantities within the $k$ th layer with $z_{k-1} \leq z \leq z_{k}$, where $z_{k-1}$ is the vertical coordinate of the bottom interface of this layer.

Using the same notation as for the RZT [18,19,21,23], we propose the following displacement field for the $k$ th layer

$$
\left\{\begin{array}{l}
u^{k}(x, z)=u_{0}(x)-z \frac{d w_{0}}{d x}+\bar{u}^{k}(x, z) \\
w(x, z)=w_{0}(x)
\end{array}\right.
$$

where $u_{0}(x)$ is the axial displacement, $w_{0}$ is the vertical deflection and $\bar{u}^{k}(x, z)$ is the zigzag displacement function that represents the distortion of the cross section relative to the plane normal to the deformed axis of the beam (Figure 1). The following expression for the zigzag function is assumed

$$
\bar{u}^{k}(x, z)=\left(\phi_{s}^{k}(z)+\phi_{d}^{k}(z)\right) \psi(x)
$$

where $\phi_{s}^{k}(z)$ takes into account the distortion effects due to shear elastic strains, $\phi_{d}^{k}(z)$ are the distortion effects due to delamination and $\psi(x)$ is the shear angle.

Function $\phi_{s}^{k}(z)$ is defined as follow

$$
\phi_{s}^{k}(z)=-z+\bar{\phi}_{s}^{k-1}+\beta_{s}^{k}\left(z-z_{k-1}\right)
$$

where the parameters $\bar{\phi}_{s}^{k-1}$ and $\beta_{s}^{k}$ are represented in Figure 2.

Function $\phi_{d}^{k}(z)$ is defined as 


$$
\phi_{d}^{k}(z)=\bar{\phi}_{d}^{k-1}+\beta_{d}\left(z-z_{k-1}\right)
$$

with $\bar{\phi}_{d}^{k-1}$ and $\beta_{d}$ expressed as

$$
\begin{gathered}
\bar{\phi}_{d}^{k-1}=\beta_{d}\left(z_{k-1}+\frac{h}{2}\right)-h\left(\sum_{i=1}^{k} \beta_{d}^{i}\right) \\
\beta_{d}=\sum_{i=1}^{n_{\text {ifaces }}} \beta_{d}^{i}
\end{gathered}
$$

where $n_{\text {ifaces }}=n_{\text {layers }}-1$ is the number of intefaces, $\beta_{d}^{i}$ is a parameter that represents the degree of delamination in the ith interface. The value of $\beta_{d}^{i}$ is considered to be known in this work and ranges between 0 (no damage) and 1 (total delamination).

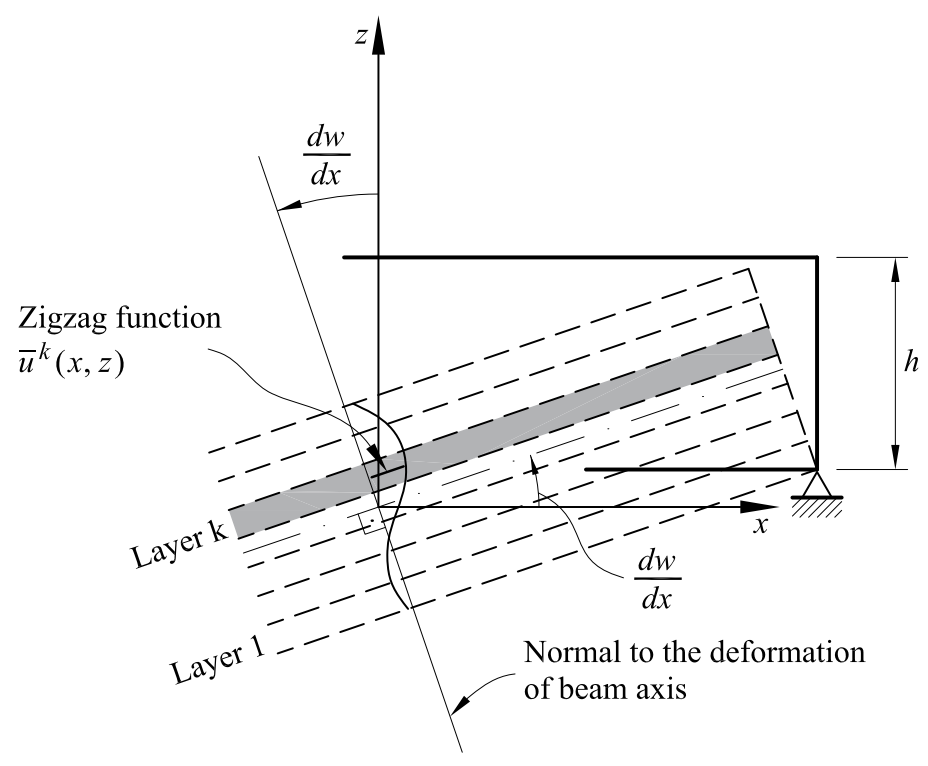

Figure 1 Transverse cross section deformation

Functions $\bar{\phi}_{s}^{k}$ and $\bar{\phi}_{d}^{k}$ satisfy the following conditions at the end fibers of the section

$$
\begin{aligned}
& \bar{\phi}_{s}^{0}=\bar{\phi}_{s}^{n}=0 \\
& \bar{\phi}_{d}^{0}=\bar{\phi}_{d}^{n}=0
\end{aligned}
$$

From Figure 2 it is deduced that $\beta_{s}^{k}$ is constant within each layer, i.e.

$$
\beta_{s}^{k}=\frac{d\left[\bar{\phi}_{s}^{k-1}+\beta_{s}^{k}\left(z-z_{k-1}\right)\right]}{d z}=\frac{\bar{\phi}_{s}^{k}-\bar{\phi}_{s}^{k-1}}{h^{k}}
$$

where $h^{k}$ is the thickness of the $k$ th layer. 


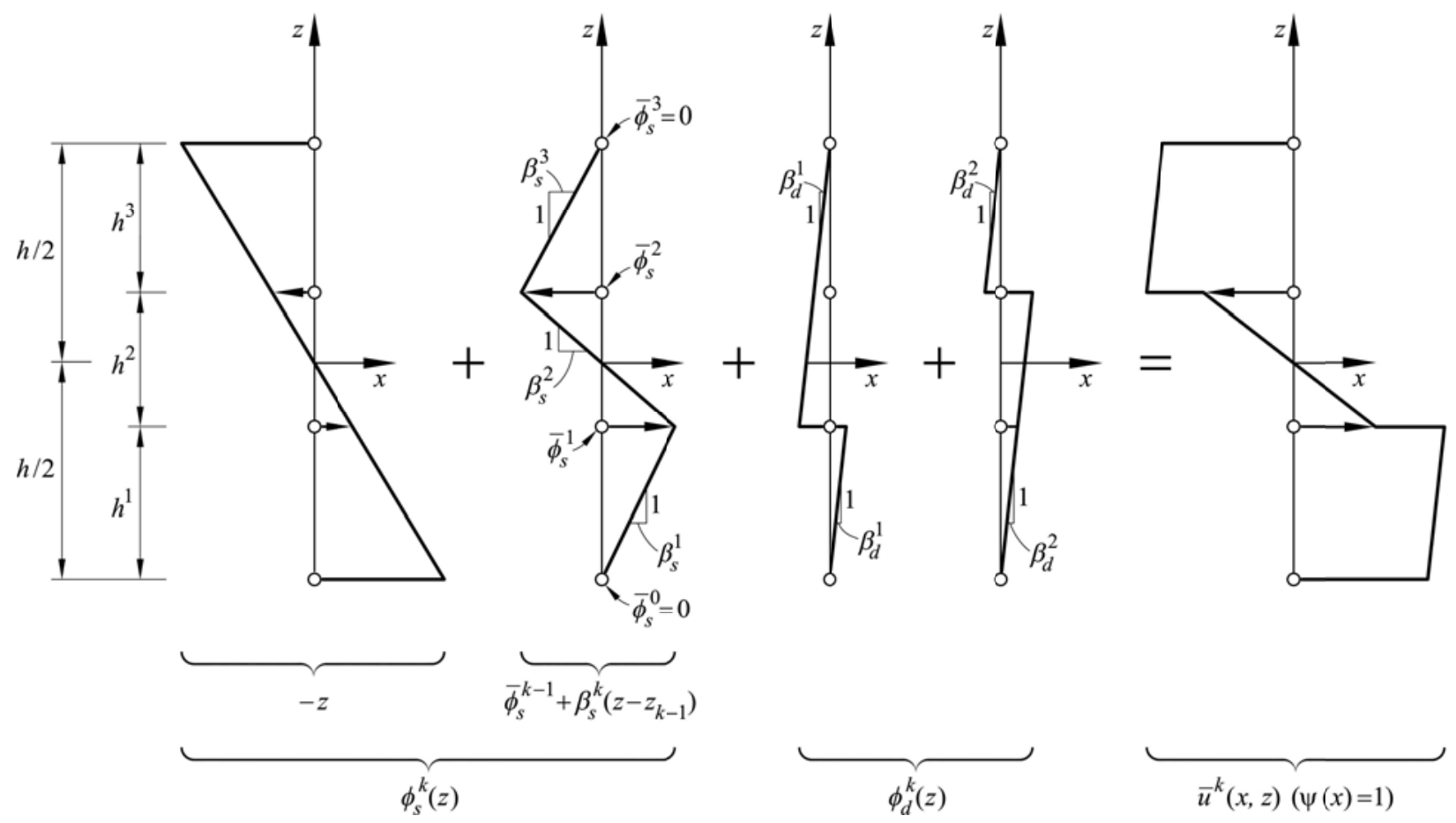

Figure 2 Definition of the zigzag function $\bar{u}^{k}(x, z)$

From Eqs. (7) and (9) we find

$$
\iint_{A} \beta_{s}^{k} d A=0
$$

\section{2-2) Strain and stress fields}

The strain-displacement relations are derived from Eqs.(1), (2) and (3) as

$$
\begin{aligned}
& \varepsilon_{x}^{k}=\frac{\partial u}{\partial x}=\frac{d u_{0}}{d x}-z \frac{d^{2} w_{0}}{d x^{2}}+\left(\phi_{s}^{k}+\phi_{d}^{k}\right) \frac{d \psi}{d x}=\underbrace{\left[\begin{array}{lll}
1 & -z & \left(\phi_{s}^{k}+\phi_{d}^{k}\right)
\end{array}\right]}_{\mathbf{S}_{u}^{k}}\left\{\begin{array}{c}
\frac{d u_{0}}{d x} \\
\frac{d^{2} w_{0}}{d x^{2}} \\
\frac{d \psi}{d x}
\end{array}\right\}=\mathbf{S}_{u}^{k} \hat{\boldsymbol{\varepsilon}}_{u} \\
& \gamma_{x z}^{k}=\frac{\partial u}{\partial z}+\frac{\partial w}{\partial x}=\underbrace{\left[-1+\beta_{s}^{k}+\beta_{d}\right]}_{\mathbf{S}_{t}^{k}}\{\psi\}=\mathbf{S}_{t}^{k} \hat{\varepsilon}_{t}
\end{aligned}
$$

where $\varepsilon_{u}^{k}$ and $\varepsilon_{t}^{k}$ are the generalized in-plane (axial-bending) and transverse shear strain vectors respectively.

Assuming that the material for the $k$ th layer is linear elastic and orthotropic with the orthotropy axes corresponding to the Cartesian coordinates $(x, z)$, the axial and shear stresses can be expressed in term of the corresponding generalized strains as 


$$
\begin{aligned}
& \sigma_{x}^{k}=E^{k} \varepsilon_{x}^{k}=E^{k} \mathbf{S}_{u}^{k} \hat{\boldsymbol{\varepsilon}}_{t} \\
& \tau_{x z}^{k}=G^{k} \gamma_{x z}^{k}=G^{k} \mathbf{S}_{t}^{k} \hat{\boldsymbol{\varepsilon}}_{t}
\end{aligned}
$$

where $E^{k}$ and $G^{k}$ are the axial and shear moduli for the $k$ th layer, respectively.

\section{Remark 1}

Different works [18-20] have shown that beam models requiring $C^{1}$ continuity for the deflection field, such as that presented in Eq.(1), have theoretical difficulties to represent correctly the shear strain and the shear stress distributions in a clamped support where the following kinematic conditions are required

$$
u_{0}=w_{0}=\frac{d w_{0}}{d x}=\psi=0
$$

Replacing condition $\psi=0$ in Eqs.(12) and (14) we find that the shear strain and the shear stress vanish in a clamped support. This situation has not a physical sense.

This apparent inconsistency of the model can be overcome if we consider that the shear strain in Eq. (14) is only a part of the total shear strain. The remainder part of the shear strain may be evidenced if we enhance the vertical deflection field $w(x, z)$ in Eq.(1) by incorporating an additional function $w_{1}(x, z)$ as

$$
w(x, z)=w_{0}(x)+w_{1}(x, z)
$$

To be consistent with the formulation, the expression of $\left.w_{1}(x, z)\right)$ in Eq (16) must be defined in a way that it not affects the vertical displacement of the beam axis, which is defined by $w_{0}(x)$. It could be defined as $w_{1}(x, z)=f_{1}(x) \cdot f_{2}(z)$, where $f_{2}(\mathrm{z})$ is prescribed function which is subjected to the restriction of $f_{2}(0)=0$. In this way $w_{1}(x, 0)=0$ and $w(x, 0)=w_{0}(x)$.

Using Eq. (16) and taking into account Eqs.(12) and (14), the total shear strains $\bar{\gamma}_{x z}^{k}$ and the total shear stresses $\bar{\tau}_{x z}^{k}$ can be written as

$$
\begin{gathered}
\bar{\gamma}_{x z}^{k}=\frac{\partial u}{\partial z}+\frac{\partial w}{\partial x}=\underbrace{\left[-1+\beta_{s}^{k}+\beta_{d}\right]}_{\mathbf{S}_{t}^{k}}\{\psi\}+\frac{\partial w_{1}}{\partial x}=\mathbf{S}_{t}^{k} \hat{\boldsymbol{\varepsilon}}_{t}+\gamma_{x z, 1}^{k} \\
\bar{\tau}_{x z}^{k}=G^{k} \gamma_{x z}^{k}=G^{k} \mathbf{S}_{t}^{k} \hat{\boldsymbol{\varepsilon}}_{t}+G^{k} \gamma_{x z, 1}^{k}
\end{gathered}
$$

The additional part of the shear strain $\gamma_{x z, 1}^{k}$ is not subjected to a vanishing condition in a clamped support. In this way the theoretical inconsistency mentioned previously is overcome.

In the present model we consider that the elastic energy related to $\gamma_{x z, 1}^{k}$ is negligible in comparison with the elastic energy related with $\mathbf{S}_{u}^{k} \hat{\boldsymbol{\varepsilon}}_{u}$ and $\mathbf{S}_{t}^{k} \hat{\boldsymbol{\varepsilon}}_{t}$. Hence 


$$
\frac{\int_{V} \frac{1}{2} G^{k}\left[\left(\gamma_{x z, 1}^{k}\right)^{2}+2 \gamma_{x z, 1}^{k}\left(\mathbf{S}_{t}^{k} \hat{\boldsymbol{\varepsilon}}_{t}\right)\right] d V}{\int_{V}\left\{\frac{1}{2} E^{k}\left[\mathbf{S}_{u}^{k} \hat{\boldsymbol{\varepsilon}}_{u}\right]^{T}\left[\mathbf{S}_{u}^{k} \hat{\boldsymbol{\varepsilon}}_{u}\right]+\frac{1}{2} G^{k}\left[\mathbf{S}_{t}^{k} \hat{\boldsymbol{\varepsilon}}_{t}\right]^{T}\left[\mathbf{S}_{t}^{k} \hat{\boldsymbol{\varepsilon}}_{t}\right]\right\} d V} \approx 0
$$

Following this assumption, we will not consider the additional shear strain $\gamma_{x z, 1}^{k}$ in our formulation. However, we should keep in mind that the shear strain $\gamma_{x z}^{k}=\mathbf{S}_{t}^{k} \hat{\varepsilon}_{t}$ is only a part of the total shear strain. Also it is important to note that the accurate thickness distribution of the shear stresses is calculated in a post-processing step in terms of the axial stresses by integrating the governing equations. This is explained in Section 3-2.

\section{2-3) Generalized constitutive relationship}

The resultant stresses are defined as

$$
\begin{aligned}
& \hat{\boldsymbol{\sigma}}_{u}=\left\{\begin{array}{c}
N \\
M \\
M_{\phi}
\end{array}\right\}=\iint_{A}\left[\mathbf{S}_{u}^{k}\right]^{T} \sigma_{x}^{k} d A \\
& \hat{\boldsymbol{\sigma}}_{t}=\left\{Q_{\phi}\right\}=\iint_{A}\left[\mathbf{S}_{t}^{k}\right]^{T} \tau_{x z}^{k} d A
\end{aligned}
$$

In above expressions, $N, M$ are, respectively, the axial force and the bending moment of the standard beam theory, whereas $M_{\phi}$ and $Q_{\phi}$ are an additional bending moment and an additional shear force which are conjugate to the new generalized strains $\frac{\partial \psi}{\partial x}$ and $\psi$, respectively.

From the relations (13) and (14) the generalized stresses can be obtained as

$$
\begin{gathered}
\hat{\boldsymbol{\sigma}}_{u}=\iint_{A}\left[\mathbf{S}_{u}^{k}\right]^{T} \sigma_{x}^{k} d A=\left(\iint_{A}\left[\mathbf{S}_{u}^{k}\right]^{T} \mathbf{S}_{u}^{k} E^{k} d A\right) \hat{\boldsymbol{\varepsilon}}_{u}=\hat{\boldsymbol{D}}_{u} \hat{\boldsymbol{\varepsilon}}_{u} \\
\hat{\boldsymbol{\sigma}}_{t}=\iint_{A}\left[\mathbf{S}_{t}^{k}\right]^{T} \tau_{x}^{k} d A=\left(\iint_{A}\left[\mathbf{S}_{t}^{k}\right]^{T} \mathbf{S}_{t}^{k} G^{k} d A\right) \hat{\boldsymbol{\varepsilon}}_{t}=\hat{\boldsymbol{D}}_{t} \hat{\boldsymbol{\varepsilon}}_{t}
\end{gathered}
$$

The generalized matrices $\hat{\mathbf{D}}_{u}$ and $\hat{\mathbf{D}}_{t}$ are

$$
\begin{gathered}
\hat{\mathbf{D}}_{u}=\iint_{A} E^{k}\left[\begin{array}{ccc}
1 & -z & \phi^{k} \\
-Z & z^{2} & -z \phi^{k} \\
\phi^{k} & -z \phi^{k} & \left(\phi^{k}\right)^{2}
\end{array}\right] d A \\
\hat{\mathbf{D}}_{t}=\left[D_{s}\right] \text { with } D_{s}=\iint_{A} G^{k}\left(-1+\beta_{s}^{k}+\beta_{d}\right)^{2} d A
\end{gathered}
$$




\section{2-4) Virtual work expression}

The virtual work expression for a vertical distributed load $q$ is

$$
\iiint_{V}\left(\delta \varepsilon_{x}^{k} \sigma_{x}^{k}+\delta \gamma_{x z}^{k} \tau_{x z}^{k}\right) d V-\int_{L} \delta w q d x=0
$$

Substituting Eqs. (11) and (12) into the LHS term of Eq.(26) and using Eqs.(20) and (21) we obtain

$$
\begin{aligned}
\iiint_{V}\left(\delta \varepsilon_{x}^{k} \sigma_{x}^{k}+\delta \gamma_{x z}^{k} \tau_{x z}^{k}\right) d V & =\iiint_{V}\left(\delta \hat{\boldsymbol{\varepsilon}}_{u}^{T}\left[\mathbf{S}_{u}^{k}\right]^{T} \sigma_{x}^{k}+\delta \hat{\boldsymbol{\varepsilon}}_{t}^{T}\left[\mathbf{S}_{t}^{k}\right]^{T} \tau_{x z}^{k}\right) d V \\
& =\int_{L}\left(\delta \hat{\boldsymbol{\varepsilon}}_{u}^{T} \hat{\boldsymbol{\sigma}}_{u}+\delta \hat{\boldsymbol{\varepsilon}}_{t}^{T} \hat{\boldsymbol{\sigma}}_{t}\right) d x
\end{aligned}
$$

The virtual work expression is therefore written as

$$
\int_{L}\left(\delta \hat{\boldsymbol{\varepsilon}}_{u}^{T} \hat{\boldsymbol{\sigma}}_{u}+\delta \hat{\boldsymbol{\varepsilon}}_{t}^{T} \hat{\boldsymbol{\sigma}}_{t}\right) d x-\int_{L} \delta w q d x=0
$$

\section{3-) EEBZ2 beam element}

\section{3-1) Formulation}

The kinematic variables are $u_{0}, w, d w / d x$ and $\psi$. The variables $u_{0}$ and $\psi$ are discretized using 2-noded linear $C^{0}$ beam elements of length $l^{(e)}$. The deflection $w$ is discretized with cubic $C^{1}$ beam elements of length $l^{(e)}$. Therefore, we can write

$$
\mathbf{u}=\left\{\begin{array}{l}
u_{0} \\
w_{0} \\
\frac{d w}{d x} \\
\psi
\end{array}\right\}=\sum_{i=1}^{2} \mathbf{N}_{i} \mathbf{a}_{i}^{(e)}=\mathbf{N a}^{(e)}
$$

with

$$
\mathbf{N}=\left[\begin{array}{ll}
\mathbf{N}_{1} & \mathbf{N}_{2}
\end{array}\right], \mathbf{N}_{i}=\left[\begin{array}{cccc}
N_{i}^{l} & 0 & 0 & 0 \\
0 & N_{i}^{c} & \bar{N}_{i}^{c} & 0 \\
0 & \frac{d N_{i}^{c}}{d x} & \frac{d \bar{N}_{i}^{c}}{d x} & 0 \\
0 & 0 & 0 & N_{i}^{l}
\end{array}\right], \mathbf{a}^{(e)}=\left\{\begin{array}{l}
\mathbf{a}_{1}^{(e)} \\
\mathbf{a}_{2}^{(e)}
\end{array}\right\}, \mathbf{a}_{i}^{(e)}=\left\{\begin{array}{c}
u_{0 i} \\
w_{0 i} \\
\frac{d w}{d x_{i}} \\
\psi_{i}
\end{array}\right\}
$$

where $N_{i}^{l}$ are the standard 1D linear shape functions and $N_{i}^{c}$ and $\bar{N}_{i}^{c}$ are the Hermite shape functions used in the classical EBT.

Substituting Eq.(29) in the generalized strain vector of Eqs.(11) and (12) gives

$$
\hat{\boldsymbol{\varepsilon}}_{u}=\mathbf{B}_{u} \mathbf{a}^{(e)}, \hat{\boldsymbol{\varepsilon}}_{t}=\mathbf{B}_{t} \mathbf{a}^{(e)}
$$


The generalized strain matrices $\mathbf{B}_{u}$ and $\mathbf{B}_{t}$ are

$$
\mathbf{B}_{u}=\left[\begin{array}{ll}
\mathbf{B}_{u 1} & \mathbf{B}_{u 2}
\end{array}\right], \quad \mathbf{B}_{t}=\left[\begin{array}{ll}
\mathbf{B}_{t 1} & \mathbf{B}_{t 2}
\end{array}\right]
$$

with

$$
\mathbf{B}_{u i}=\left[\begin{array}{llll}
\frac{d N_{i}^{l}}{d x} & 0 & 0 & 0 \\
0 & \frac{d^{2} N_{i}^{c}}{d x^{2}} & \frac{l^{(e)}}{2} \frac{d^{2} \bar{N}_{i}^{c}}{d x^{2}} & 0 \\
0 & 0 & 0 & \frac{d N_{i}^{l}}{d x}
\end{array}\right] \quad, \quad \mathbf{B}_{t i}=\left[\begin{array}{llll}
0 & 0 & 0 & N_{i}^{l}
\end{array}\right]
$$

where $\mathbf{B}_{u i}$ and $\mathbf{B}_{t i}$ are the in-plane and transverse shear strain matrices for node $i$.

The virtual displacement and the generalized strain fields are expressed in terms of the virtual nodal DOFs as

$$
\delta \mathbf{u}=\mathbf{N} \delta \mathbf{a}^{(e)}, \delta \hat{\boldsymbol{\varepsilon}}_{u}=\mathbf{B}_{u} \delta \mathbf{a}^{(e)}, \delta \hat{\boldsymbol{\varepsilon}}_{t}=\mathbf{B}_{t} \delta \mathbf{a}^{(e)}
$$

Substituting Eqs.(20), (21), (29), (34), (31) and (34) into the virtual work expression (28), and following the classical procedure in the finite element method the discretized equilibrium equations are obtained as

$$
\mathbf{K a}-\mathbf{f}=\mathbf{0}
$$

where $\mathbf{K}$ is the global stiffness matrix, $\mathbf{a}$ is the vector of nodal DOFs for the whole mesh and $\mathbf{f}$ is the equivalent force vectors. Matrix $\mathbf{K}$ and vector $\mathbf{f}$ are assembled from the element contributions given by

$$
\mathbf{K}^{(e)}=\int_{l^{(e)}}\left(\mathbf{B}_{u}^{T} \hat{\mathbf{D}}_{u} \mathbf{B}_{u}+\mathbf{B}_{t}^{T} \hat{\mathbf{D}}_{t} \mathbf{B}_{t}\right) d x, \mathbf{f}^{(e)}=\int_{l^{(e)}} \mathbf{N}^{T} \mathbf{q} d x=
$$

with $\mathbf{q}=\left[\begin{array}{llll}0 & q & 0 & 0\end{array}\right]^{T}$.

The new beam element is termed EEBZ2 (for 2-noded Extended Euler-Bernoulli beam element based on the Zig-zag theory).

\section{3-2) Calculation of the thickness distribution of the shear stress}

An accurate thickness distribution of the shear stress $\tau_{x z}^{k}$ can be calculated starting from the Cauchy equilibrium equations (Figure 3 )

$$
\frac{\partial \sigma_{x}^{k}}{\partial x}+\frac{\partial \bar{\tau}_{x z}^{k}}{\partial z}=0 \Rightarrow \frac{\partial \bar{\tau}_{x z}^{k}}{\partial z}=-\frac{\partial \sigma_{x}^{k}}{\partial x}
$$

Using the notation of Remark 1, the bar in $\bar{\tau}_{x z}^{k}$ means that it is the total shear stress 
From Eq.(37) we deduce

$$
\bar{\tau}_{x z}^{k}=\int_{z_{k}}^{h / 2}-\frac{\partial \sigma_{x}^{k}}{\partial x} d z=\int_{-h / 2}^{z}-\frac{\partial \sigma_{x}^{k}}{\partial x} d z
$$
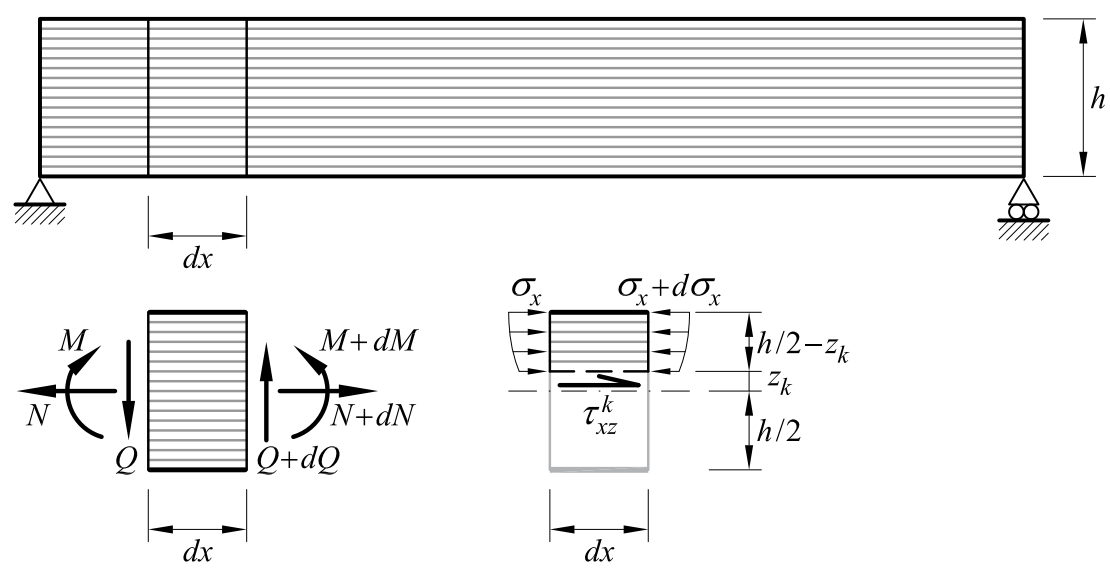

Figure 3 Sectional equilibrium

The derivative of the axial stress $\frac{\partial \sigma_{x}^{k}}{\partial x}$ can be calculated taking into account Eqs.(11) and (13). Then we have

$$
\frac{\partial \sigma_{x}^{k}}{\partial x}=E^{k} \frac{d^{2} u_{0}}{d x^{2}}-E^{k} z \frac{d^{3} w_{0}}{d x^{3}}+E^{k}\left(\phi_{s}^{k}(z)+\phi_{d}^{k}(z)\right) \frac{d^{2} \psi}{d x^{2}}
$$

Because $u_{0}(x)$ and $\psi(x)$ vary linearly within an element the second derivatives of these functions would be null. A linear variation for the first derivative of these functions can be approximated with the following expressions

$$
\begin{aligned}
& \frac{\overline{d u_{0}}}{d x}=N_{1}^{l}(x) \frac{\overline{d u_{01}}}{d x}+N_{2}^{l}(x) \frac{\overline{d u_{02}}}{d x} \\
& \frac{\overline{d \psi}}{d x}=N_{1}^{l}(x) \frac{\overline{d \psi_{1}}}{d x}+N_{2}^{l}(x) \frac{\overline{d \psi_{2}}}{d x}
\end{aligned}
$$

where $\frac{\overline{d u_{01}}}{d x}, \frac{\overline{d u_{02}}}{d x}, \frac{\overline{d \psi_{1}}}{d x}$ and $\frac{\overline{d \psi_{2}}}{d x}$ are smoothed nodal values computed by averaging the values contributed by the elements adjacent to the node. In Figure 4 is described the procedure for obtaining the smoothed nodal values $\frac{\overline{d \psi_{1}}}{d x}$ and $\frac{\overline{d \psi_{2}}}{d x}$. Similar procedure is applied for obtaining $\overline{\frac{d u_{01}}{d x}}$ and $\frac{\overline{d u_{02}}}{d x}$.

Taking the derivative of each side of Eqs.(40) and (41) an approximate expression for the second derivatives in Eq. (39) can be obtained 


$$
\begin{aligned}
& \frac{d^{2} u_{0}}{d x^{2}}=\frac{d N_{1}^{l}}{d x} \frac{\overline{d u_{01}}}{d x}+\frac{d N_{2}^{l}}{d x} \frac{\overline{d u_{02}}}{d x} \\
& \frac{d^{2} \psi}{d x^{2}}=\frac{d N_{1}^{l}}{d x} \frac{\overline{d \psi_{1}}}{d x}+\frac{d N_{2}^{l}}{d x} \frac{\overline{d \psi_{2}}}{d x}
\end{aligned}
$$

Substituting Eq.(39) in (38) yields the following expression for the total shear stress $\bar{\tau}_{x z}^{k}$

$$
\bar{\tau}_{x z}^{k}=-\left(\int_{-h / 2}^{z} E^{k} d z\right) \frac{d^{2} u_{0}}{d x^{2}}+\left(\int_{-h / 2}^{z} E^{k} z d z\right) \frac{d^{3} w_{0}}{d x^{3}}-\left(\int_{-h / 2}^{z} E^{k}\left(\phi_{s}^{k}(z)+\phi_{d}^{k}(z)\right) d z\right) \frac{d^{2} \psi}{d x^{2}}
$$
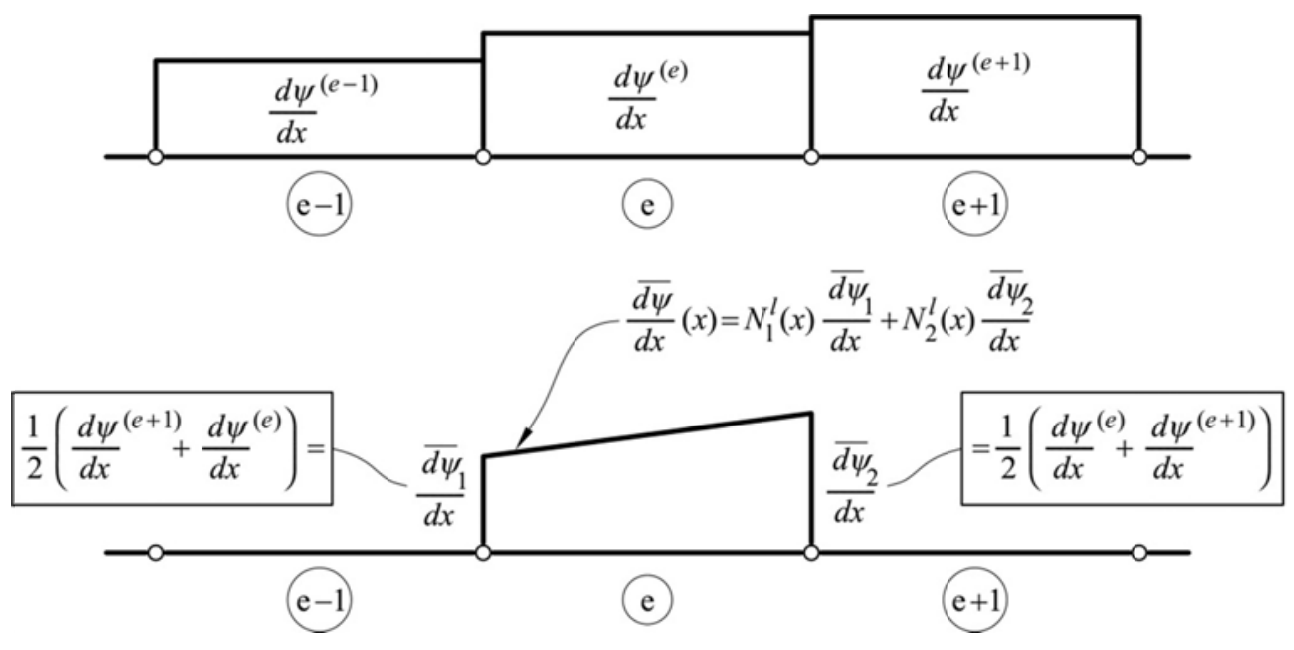

Figure 4 Averaging procedure for the smoothed nodal values $\frac{\overline{d \psi_{1}}}{d x}$ and $\frac{\overline{d \psi_{2}}}{d x}$.

\section{4-) Computation of the parameter $\beta_{s}^{k}$}

To define completely the model is necessary to compute the parameter $\beta_{s}^{k}$. Depending on the characteristics of the laminate we can consider two situations.

First we consider laminated beams having layers with similar mechanical properties. In this case the distortion effects due to shear elastic strains are principally relevant for low values of the span-to-thickness ratio of the beam. A very good behavior of the whole range of beam slenderness can be obtained if in this case, the zigzag function is used to approximate better the distribution of the shear strain in the cross section.

Secondly, we consider laminated beams having layers with different mechanical properties. Conversely to the previous situation, in this case the distortion effects due to shear elastic strains can be of great relevance for any value of the span-to-thickness ratio of the beam. In order to obtain an accurate global response of the model, we need to adequately reproduce the distortion effects in the layers at a section level. For this reason, a constant thickness distribution of the 
shear strain is considered for computing $\beta_{s}^{k}$. This takes into account the distortion effects in the layers in an average sense.

The two situations commented above are detailed in the next sections.

\section{4-1) Laminated beams having layers with similar mechanical properties}

In this case the delamination effects are neglected, i.e. $\beta_{d}=0$. Substituting Eq.(12) into Eq.(14), the shear stress in the $k$ th layer is computed as

$$
\tau_{x z}^{k}=G^{k}\left(-1+\beta_{s}^{k}\right) \psi
$$

Taking into account the comments of Remark 1, for a cross section far enough of a clamped support, the shear stress $\tau_{x z}^{k}$ in Eq.(45) tends to be equivalent with the total the shear stress $\bar{\tau}_{x z}^{k}$ in Eq.(44). However, in the case of laminate beams having layers with similar mechanical properties the influence over the resultant shear stress of first and third terms of the right hand side of Eq.(44), can be considered negligible in comparison with the second one. Then considering only the term related to the third derivative of $w_{0}(x)$, we can write

$$
\tau_{x z}^{k}=\left(\int_{-h / 2}^{z_{k}} E^{k} z d z\right) \frac{d^{3} w_{0}}{d x^{3}}=S_{E}^{k} \frac{d^{3} w_{0}}{d x^{3}}
$$

with

$$
S_{E}^{k}(z)=\sum_{i=1}^{k-1}\left[\frac{E^{i}}{2}\left(z_{i}^{2}-z_{i-1}^{2}\right)\right]+\frac{E^{k}}{2}\left(z^{2}-z_{k-1}^{2}\right)
$$

Equaling Eqs.(45) and Eqs (46) the following expression is obtained

$$
\left(-1+\beta_{s}^{k}\right) \psi=\frac{S_{E}^{k}}{G^{k}} \frac{d^{3} w_{0}}{d x^{3}}
$$

Integrating Eq.(48) over the area the and taking into account Eq.(10) yields

$$
-A \psi=I_{q} \frac{d^{3} w_{0}}{d x^{3}} \Rightarrow \psi=-\frac{I_{q}}{A} \frac{d^{3} w_{0}}{d x^{3}}
$$

with

$$
\begin{aligned}
& I_{q}=\int_{-h / 2}^{h / 2} \frac{S_{E}^{k}(z) b^{k}}{G^{k}} d z= \\
& \sum_{k=1}^{n_{\text {layers }}}\left[\frac{h^{k} b^{k}}{G^{k}}\left(\sum_{i=1}^{k-1} \frac{E^{i}}{2}\left(z_{i}^{2}-z_{i-1}^{2}\right)\right)+\frac{E^{k} b^{k}}{2 G^{k}}\left(\frac{\left(z_{k}^{3}-z_{k-1}^{3}\right)}{3}-z_{k-1}^{2} h^{k}\right)\right]
\end{aligned}
$$

Replacing Eq.(49) into Eq.(48) yields 


$$
\beta_{s}^{k}=\left(1-\frac{S_{E}^{k} A}{I_{q} G^{k}}\right)
$$

\section{4-2) Laminated beams having layers with different mechanical properties}

As we have previously explained, a constant shear stress distribution is assumed over the whole cross section. From Eq.(12)

$$
\tau_{x z}^{k}=G^{k}\left(-1+\beta_{s}^{k}+\beta_{d}\right) \psi=c t e \quad \forall k
$$

where cte means that all the layers have the same constant value for the shear stress.

Condition (52) can be enforced by constraining the term multiplying $\psi$ to be constant, i.e.

$$
G^{k}\left(-1+\beta_{s}^{k}+\beta_{d}\right)=G^{k+1}\left(-1+\beta_{s}^{k+1}+\beta_{d}\right)=-G=\text { cte } \quad \forall k
$$

From Eq.(53) it can be deduced

$$
\beta_{s}^{k}=1-\beta_{d}-\frac{G}{G^{k}}
$$

Substituting $\beta_{s}^{k}$ in the integral of Eq.(10) gives

$$
\sum_{k=1}^{n_{\text {lavers }}}\left[\left(1-\beta_{d}\right) h^{k}-\frac{G}{G^{k}} h^{k}\right]=0
$$

Isolating the variable $\mathrm{G}$ from Eq.(55) gives

$$
G=\left(1-\beta_{d}\right) h\left[\sum_{k=1}^{n_{\text {layers }}} \frac{h^{k}}{G^{k}}\right]^{-1}
$$

In order to avoid numerical ill-conditioning in the stiffness matrix Eq.(56) is substituted by

$$
G=\operatorname{Max}\left[\left\langle 1-\beta_{d}\right\rangle h\left[\sum_{k=1}^{n_{\text {layers }}} \frac{h^{k}}{G^{k}}\right]^{-1}, G_{\min }\right]
$$

which is the equivalent shear modulus for the laminate. The symbol $\langle\bullet\rangle$ is the Macaulay parenthesis and is used to avoid negative values of $G . G_{\min }$ is the assumed residual value for $G$. Replacing Eq.(54) in Eq.(52) yields

$$
\tau_{x z}^{k}=-G \psi=c t e \quad \forall k
$$




\section{5-) Analysis of the behavior of the EEBZ2 element for the whole range of the beam slenderness}

\section{5-1) Introduction}

The stiffness matrix and the force vector of Eq. (35) are obtained in closed form by exact integration. This characteristic of the EEBZ2 element is in contrast the zigzag beam elements presented in [20-23] which require reduced integration of some stiffness matrix terms to avoid shear locking.

The performance of the EEBZ2 beam element is evaluated in the analysis of a cantilever beam and a simple supported beam with different span-to-thickness ratios $\lambda=L / h$. We consider laminated beams that have layers with similar mechanical properties and, therefore, the zigzag function proposed in Section 4-1 is used.

For comparison purposes the same beams are analyzed using meshes of four-noded plane stress rectangles which results are taken as reference values.

Both beams are analyzed also with meshes of two-noded beam elemens based on the classical TBT. In all TBT results presented in this paper a shear correction factor of $5 / 6$ is considered.

\section{5-2) Cantilever beam}

We analyze first a cantilever beam of length $L$ under an end point load of value $P=1 \mathrm{kN}$ (Figure 4$)$. The beam has a rectangular section $(0.04 \mathrm{~m} \times 0.02 \mathrm{~m})$ formed by ten layers. The material properties are assumed to be the same for all layers and are given in Figure 5. The analysis is performed for fourteen span-to-thickness ratios $\lambda=L / h=1,2,3,4,5,6,7,8,9,10,25,50,75,100$. We have used meshes of 3,5 and 10 EEBZ2 elements. The same beam is analyzed using meshes of four-noded plane stress square elements with a size of $5.0 \mathrm{e}-4 \mathrm{~m}$. The beam is also analyzed with a mesh of 3 two-noded TBT linear element.

The maximum deflection obtained in every analysis is normalized with the maximum analytical deflection given by the EBT as

$$
r_{w}^{i}=\frac{w_{\max }^{i}}{w_{\max }^{E B}} \quad \text { with } w_{\max }^{E B}=\frac{P L^{3}}{3 E I} \text { and } i=P S, 3,5,10 \text { and TBT-3 }
$$

where index $i$ denotes the type of solution: $i=P S$ for plane stress; $i=3,5$ or 10 for meshes of three, five and ten EBBZ2 elements, respectively, and $i=$ TBT-3 for a mesh of three two-noded TBT linear elements.

Table 1 shows a comparison of the values of $r_{w}$ obtained with the different analyses and the error considering the plane stress solution as the reference value. The error is calculated with the following expression

$$
\text { Error }^{i}=\frac{w_{\max }^{i}-w_{\max }^{P S}}{w_{\max }^{P S}} \text { with } i=3,5,10 \text { and TBT-3 }
$$

Figure 6 shows the change of $r_{w}$ with the beam slenderness ratio $\lambda=L / h$. 


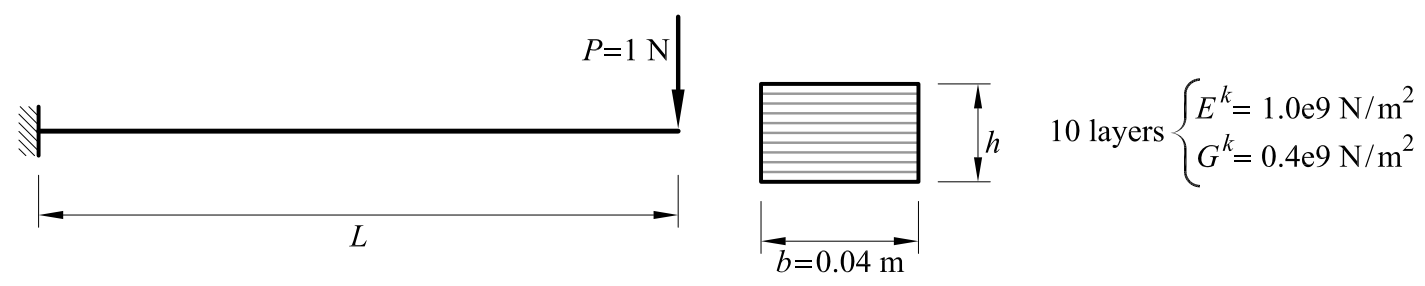

Figure 5 Cantilever beam under end point load

\begin{tabular}{|c|c|c|c|c|c|c|c|c|c|}
\hline$\lambda=\frac{L}{h}$ & $r_{w}^{P S}$ & $r_{w}^{10}$ & $\begin{array}{c}\text { Error }^{10} \\
{[\%]}\end{array}$ & $r_{w}^{5}$ & $\begin{array}{c}\text { Error }^{5} \\
{[\%]}\end{array}$ & $r_{w}^{3}$ & $\begin{array}{c}\text { Error }^{3} \\
{[\%]}\end{array}$ & $r_{w}^{\text {TBT-3 }}$ & $\begin{array}{c}\text { Error }^{\text {TBT-3 }} \\
{[\%]}\end{array}$ \\
\hline 1 & 1.684 & 1.698 & -0.84 & 1.685 & -0.07 & 1.662 & 1.31 & 1.722 & -2.27 \\
\hline 2 & 1.173 & 1.179 & -0.47 & 1.174 & -0.08 & 1.167 & 0.49 & 1.160 & 1.14 \\
\hline 3 & 1.076 & 1.080 & -0.33 & 1.078 & -0.13 & 1.075 & 0.16 & 1.056 & 1.92 \\
\hline 4 & 1.042 & 1.045 & -0.27 & 1.044 & -0.14 & 1.042 & 0.03 & 1.019 & 2.22 \\
\hline 5 & 1.027 & 1.029 & -0.22 & 1.028 & -0.14 & 1.027 & -0.03 & 1.002 & 2.37 \\
\hline 6 & 1.018 & 1.020 & -0.20 & 1.019 & -0.14 & 1.019 & -0.06 & 0.993 & 2.46 \\
\hline 7 & 1.013 & 1.015 & -0.17 & 1.014 & -0.13 & 1.014 & -0.07 & 0.988 & 2.51 \\
\hline 8 & 1.010 & 1.011 & -0.14 & 1.011 & -0.12 & 1.011 & -0.08 & 0.984 & 2.55 \\
\hline 9 & 1.007 & 1.009 & -0.15 & 1.009 & -0.12 & 1.008 & -0.08 & 0.981 & 2.58 \\
\hline 10 & 1.006 & 1.007 & -0.13 & 1.007 & -0.11 & 1.007 & -0.08 & 0.978 & 2.73 \\
\hline 25 & 0.999 & 1.001 & -0.20 & 1.001 & -0.20 & 1.001 & -0.20 & 0.973 & 2.59 \\
\hline 50 & 0.999 & 1.000 & -0.14 & 1.000 & -0.14 & 1.000 & -0.14 & 0.972 & 2.64 \\
\hline 75 & 0.999 & 1.000 & -0.44 & 1.000 & -0.44 & 1.000 & -0.44 & 0.972 & 2.36 \\
\hline 100 & 0.999 & 1.000 & -0.43 & 1.000 & -0.43 & 1.000 & -0.43 & 0.972 & 2.36 \\
\hline
\end{tabular}

Table 1 Analysis of cantilever beam under end point load with the EEBZ2 beam element. 


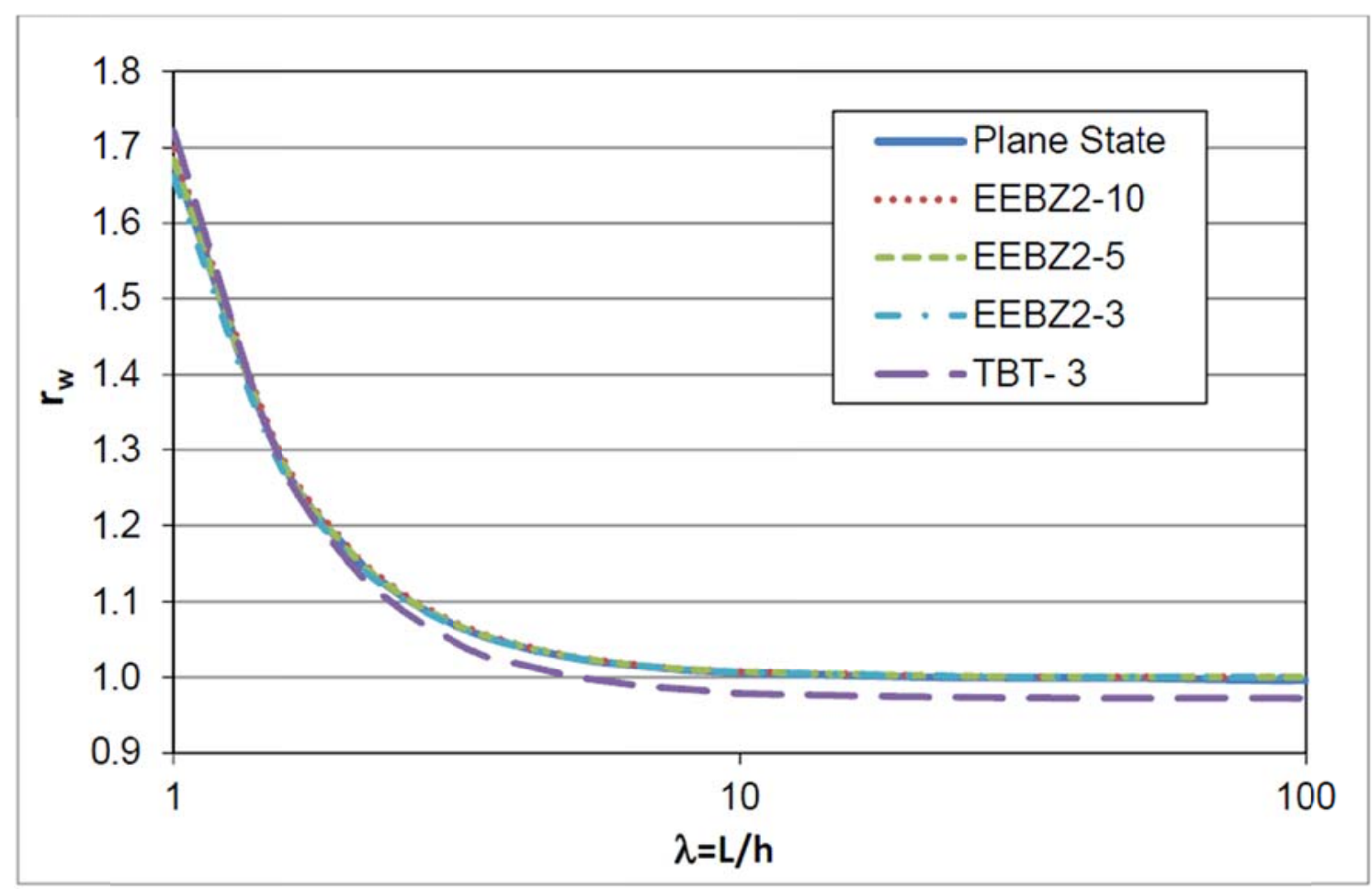

Figure 6 Cantilever beam analyzed with the EBBZ2 beam element.

The excellent performance of the EEBZ2 element for the whole range of beam slenderness can be observed.

\section{5-3) Simple supported beam}

In this example we analyze a simply supported beam of length $L$ under a uniform load of value $q=1 \mathrm{MN} / \mathrm{m}$ (Figure 7). The beam has a rectangular section $(0.04 \mathrm{~m} \times 0.02 \mathrm{~m})$ formed by ten layers. The analysis is performed for thirteen span-to-thickness ratios $\lambda=L / h=2,3,4,5,6,7,8,9,10,25,50,75,100$. We have used meshes of 2,4 and 10 of EEBZ2 elements. The same beam is analyzed using meshes of four-noded plane stress rectangles with an element size of $5.0 \mathrm{e}-4 \mathrm{~m}$. The beam is also analyzed with a mesh of 10 two-noded TBT linear beam elements.

The maximum deflection obtained in every analysis is normalized with the maximum analytical deflection given by the EBT as

$$
r_{w}^{i}=\frac{w_{\max }^{i}}{w_{\max }^{E B}} \quad \text { with } w_{\max }^{E B}=\frac{5 q L^{4}}{384 E I} \text { and } i=P S, 2,4,10 \text { and TBT-10 }
$$

where index $i$ denotes the type of solution: $i=P S$ for plane stress; $i=2,4$ or 10 for meshes of two, four and ten EBBZ2 elements, respectively, and $i=$ TBT-10 for a mesh of ten two-noded TBT linear elements.

Table 2 shows a comparison of the values of $r_{w}$ and the respective errors obtained with the different analysis versus the plane stress solution. The error is calculated with the following expression 


$$
\text { Error }^{i}=\frac{w_{\max }^{i}-w_{\max }^{P S}}{w_{\max }^{P S}} \text { with } i=2,4,10 \text { and TBT-10 }
$$

Figure 8 shows the change of $r_{w}$ with the slenderness ratio $\lambda=L / h$.

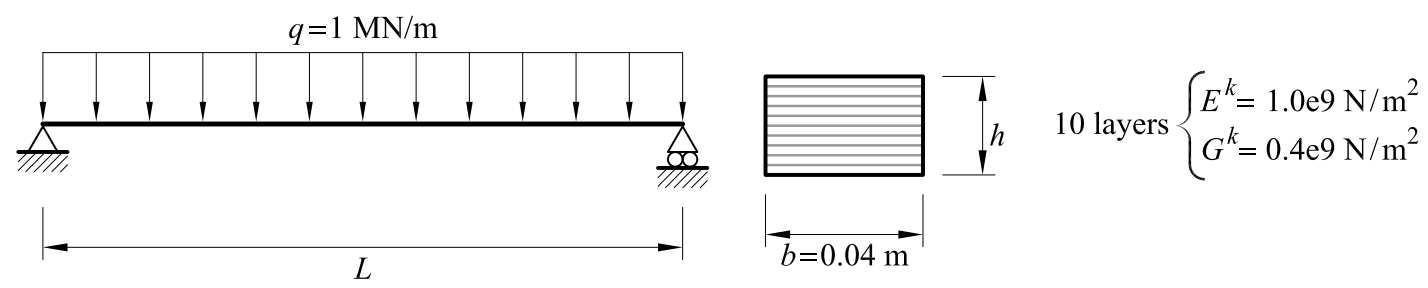

Figure 7 Simple supported beam

\begin{tabular}{|c|c|c|c|c|c|c|c|c|c|}
\hline$\lambda=\frac{L}{h}$ & $r_{w}^{P S}$ & $r_{w}^{10}$ & $\begin{array}{c}\text { Error }^{10} \\
{[\%]}\end{array}$ & $r_{w}^{4}$ & $\begin{array}{c}\text { Error }^{4} \\
{[\%]}\end{array}$ & $r_{w}^{2}$ & $\begin{array}{c}\text { Error }^{2} \\
{[\%]}\end{array}$ & $r_{w}^{\text {TBT-10 }}$ & $\begin{array}{c}\text { Error }^{\text {TBT-10 }} \\
{[\%]}\end{array}$ \\
\hline 2 & 1.537 & 1.592 & -3.57 & 1.592 & -3.60 & 1.5898 & -3.46 & 1.584 & -3.08 \\
\hline 3 & 1.242 & 1.264 & -1.73 & 1.264 & -1.74 & 1.2634 & -1.70 & 1.251 & -0.67 \\
\hline 4 & 1.137 & 1.149 & -0.99 & 1.149 & -0.99 & 1.1484 & -0.98 & 1.134 & 0.29 \\
\hline 5 & 1.088 & 1.095 & -0.66 & 1.095 & -0.66 & 1.0951 & -0.65 & 1.080 & 0.73 \\
\hline 6 & 1.061 & 1.066 & -0.47 & 1.066 & -0.47 & 1.0661 & -0.47 & 1.051 & 0.99 \\
\hline 7 & 1.045 & 1.049 & -0.36 & 1.049 & -0.36 & 1.0485 & -0.35 & 1.033 & 1.14 \\
\hline 8 & 1.034 & 1.037 & -0.28 & 1.037 & -0.28 & 1.0372 & -0.28 & 1.021 & 1.24 \\
\hline 9 & 1.027 & 1.029 & -0.23 & 1.029 & -0.21 & 1.0294 & -0.23 & 1.014 & 1.31 \\
\hline 10 & 1.022 & 1.024 & -0.19 & 1.024 & -0.19 & 1.0238 & -0.19 & 1.005 & 1.67 \\
\hline 25 & 1.002 & 1.004 & -0.19 & 1.004 & -0.19 & 1.0038 & -0.19 & 0.988 & 1.41 \\
\hline 50 & 0.995 & 1.001 & -0.64 & 1.001 & -0.64 & 1.0010 & -0.64 & 0.985 & 0.97 \\
\hline 75 & 0.996 & 1.000 & -0.42 & 1.000 & -0.42 & 1.0004 & -0.42 & 0.984 & 1.19 \\
\hline 100 & 0.999 & 1.000 & -0.73 & 1.000 & -0.73 & 1.0002 & -0.73 & 0.984 & 0.88 \\
\hline
\end{tabular}

Table 2 Comparison table for the analysis of simply supported beam with the EEBZ2 beam element. 


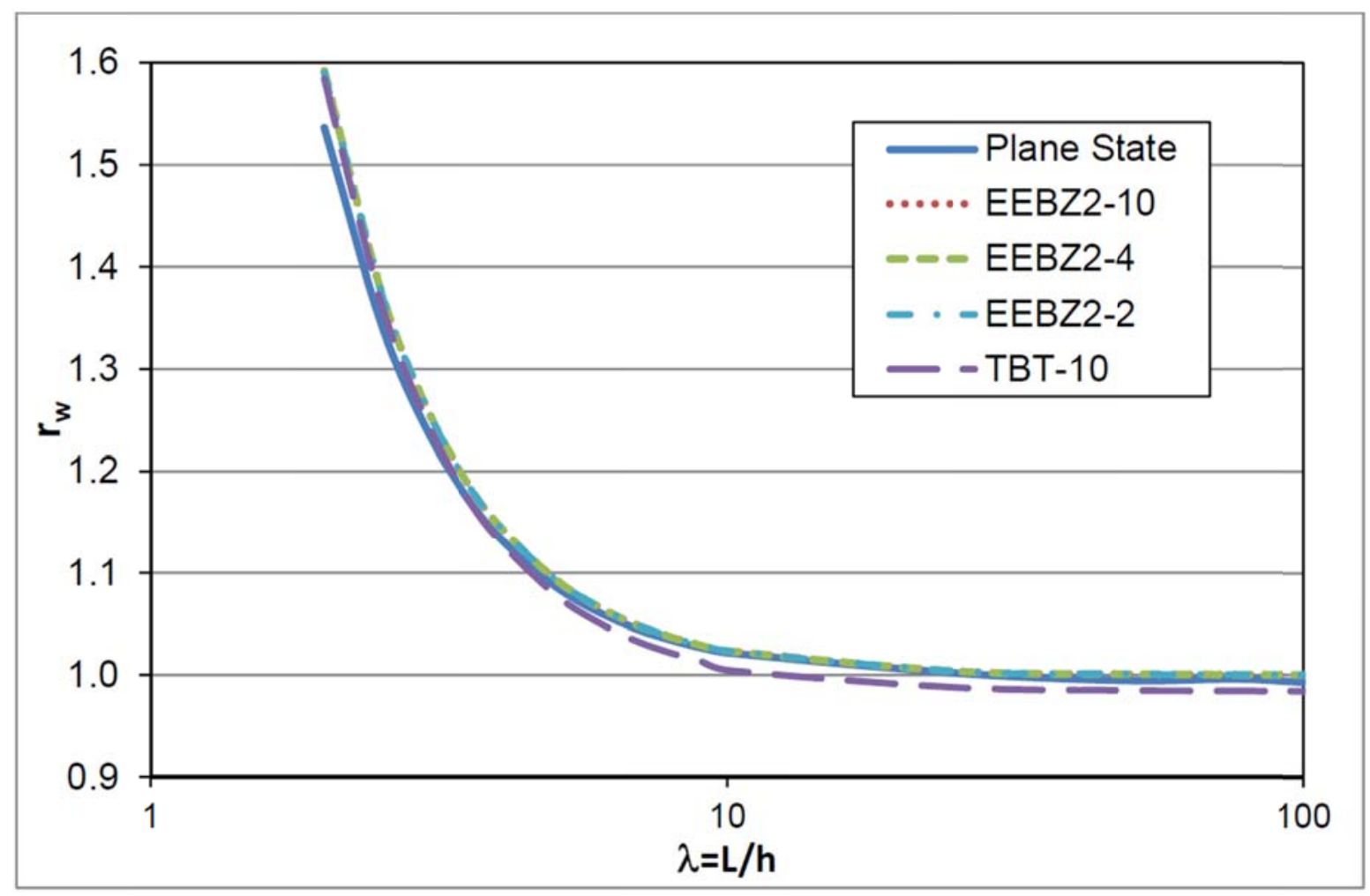

Figure 8 Simply supported beam analyzed with the EEBZ2 beam element.

An excellent performance of the EEBZ2 element formulation can be observed for the whole range of the beam slenderness. The accuracy of the element is higher for slenderness ratio values greater than five, which correspond with the typical design relations.

\section{6-) Analysis of composites laminated beams}

We present a set of application examples of laminated beams with layers with very different mechanical properties. The zigzag function proposed in Section 4-2 is used.

In order to compare the performance of the EEBZ2 beam element with other existing beam model for laminated beams we have analyzed similar examples to those presented in [21] and [37].

\section{6-1) Antisymmetric cross-ply laminated beams}

In this example a simply supported cross-ply laminated beam is analyzed. A square cross-section of side $h$ is considered. The analysis is performed for three span-to-thickness ratios $\lambda=L / h=4,10$ and 100 . An antisymmetric $\left(90^{\circ}, 0^{\circ}\right)$ staking sequence is considered. The beam is subjected to a sinusoidal load $q_{z}(x)=q_{z 0} \sin (\pi x / L)$. The two layers are assumed to be of the same thickness and the material properties adopted are

$$
\frac{E_{L}}{E_{T}}=25 \quad \frac{G_{T T}}{E_{T}}=0.2 \quad \frac{G_{L T}}{E_{T}}=0.5 \quad v_{L T}=v_{T T}=0.25
$$


where $L$ refers to the fiber direction and $T$ refers to the normal direction.

The beam has been analyzed with a mesh of 50 EEBZ2 elements which are denoted as EEBZ250. For convenience, the results are presented in non-dimensional form.

$$
\tilde{w}=100 \frac{E_{T} h^{3}}{q_{z 0} L^{4}} w, \quad \tilde{\sigma}_{x}=\frac{\sigma_{x}}{q_{z 0}}, \quad \tilde{\tau}_{x y}=\frac{\tau_{x y}}{q_{z 0}}
$$

Tables 3 to 5 show respectively the non-dimensional values of the vertical displacement at $(L / 2,0)$, axial stresses at $(L / 2, \pm h / 2)$ and tangential stress at $(0, h / 4)$. For validation purpose, results are compared with the cylindrical bending solution of Pagano [38].

\begin{tabular}{|c|c|c|c|c|}
\hline \multirow{2}{*}{ Result } & \multirow{3}{*}{ Formulation } & \multicolumn{3}{|c|}{$\lambda=L / h$} \\
\cline { 3 - 5 } & & 4 & 10 & 100 \\
\hline \multirow{2}{*}{$\tilde{w}(L / 2,0)$} & Pagano [38] & 4.67 & 2.95 & 2.62 \\
\cline { 2 - 5 } & EEBZ2-50 & 3.74 & 2.81 & 2.62 \\
\hline
\end{tabular}

Table 3 Non-dimensional vertical displacement at $x=L / 2$ and $z=0$.

\begin{tabular}{|c|c|c|c|c|}
\hline \multirow{2}{*}{ Result } & \multirow{3}{*}{ Formulation } & \multicolumn{3}{|c|}{$\lambda=L / h$} \\
\cline { 3 - 5 } & & 4 & 10 & 100 \\
\hline \multirow{2}{*}{$\left.\tilde{\sigma}_{x}(L / 2,-h / 2)\right)$} & Pagano [38] & -3.84 & -19.83 & -1894.40 \\
\cline { 2 - 5 } & EEBZ2-50 & -2.28 & -18.15 & -1891.87 \\
\hline \multirow{2}{*}{$\tilde{\sigma}_{x}(L / 2, h / 2)$} & Pagano [38] & 30.03 & 176.52 & 17442.0 \\
\cline { 2 - 5 } & EEBZ2-50 & 29.92 & 176.42 & 17434.40 \\
\hline
\end{tabular}

Table 4 Non-dimensional axial stresses at $x=L / 2$ and $z= \pm h / 2$.

\begin{tabular}{|c|c|c|c|c|}
\hline \multirow{2}{*}{ Result } & \multirow{3}{*}{ Formulation } & \multicolumn{3}{|c|}{$\lambda=L / h$} \\
\cline { 3 - 5 } & & 4 & 10 & 100 \\
\hline \multirow{2}{*}{$\tilde{\tau}_{x y}(0, h / 4)$} & Pagano [38] & 2.71 & 7.23 & 73.37 \\
\cline { 3 - 5 } & EEBZ2-50 & 3.08 & 7.37 & 72.99 \\
\hline
\end{tabular}

Table 5 Non-dimensional tangential stress at $x=L / 2$ and $z=h / 4$. 
Excellent results were obtained with the EEBZ2-50 mesh for $\lambda=10$ and 100 .

Less accurate result were obtained in the case of very low values of the span-to-thickness ratio $(\lambda=4)$. However the maximal axial and tangential stresses obtained with the EEBZ2-50 mesh have a good agreement with the reference values of Pagano [38].

In the reference [37] is presented the same example but compared with a number of other laminated beams formulation found in the literature.

\section{6-2) Three layered laminated thick cantilever beam under point load}

In this example a sandwich cantilever beam under an end-point load is analyzed (Figure 9). The material properties are given in Table 3 . The span-to-thickness ratio is $\lambda=5$.

The stiffness of the core is three orders of magnitude lower than the bottom layer. The bottom layer and the upper layer have different mechanical properties. The correct modeling of the behavior of a laminate sandwich with layers with different mechanical properties and not arranged symmetrically is a challenge for the EEBZ2 element.
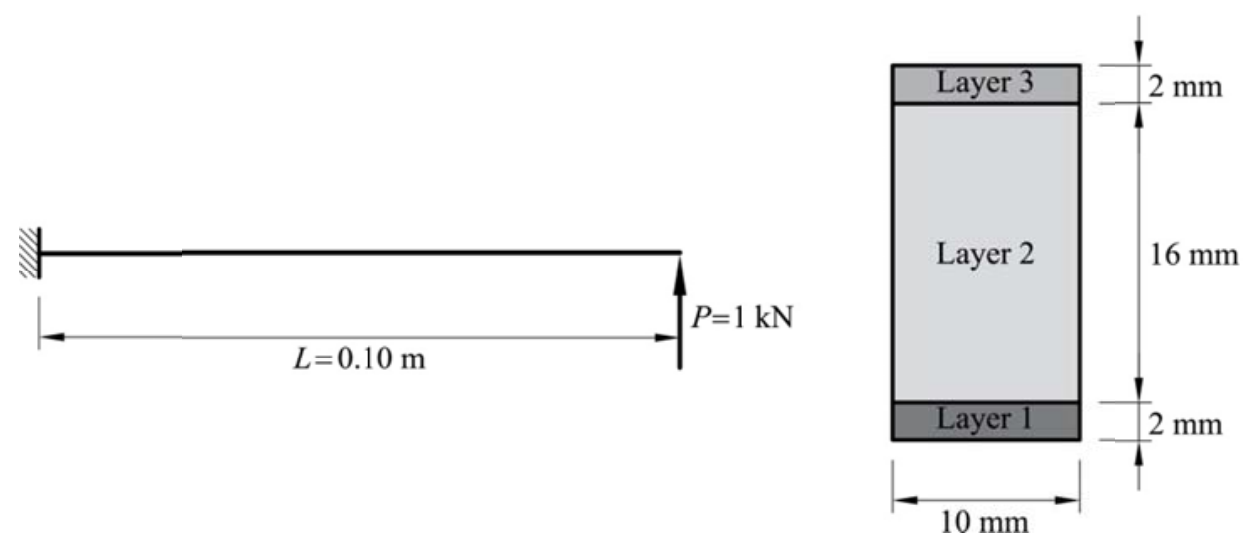

Figure 9 Three-layered laminated thick cantilever beam under end point load.

\begin{tabular}{|c|c|c|c|}
\cline { 2 - 4 } \multicolumn{1}{c|}{} & Layer 1 & Layer 2 & Layer 3 \\
\hline $\mathrm{h}[\mathrm{mm}]$ & 2 & 16 & 2 \\
\hline $\mathrm{E}[\mathrm{MPa}]$ & $7.30 \mathrm{e} 5$ & $7.30 \mathrm{e} 2$ & $2.19 \mathrm{e} 5$ \\
\hline $\mathrm{G}[\mathrm{MPa}]$ & $2.92 \mathrm{e} 5$ & $2.92 \mathrm{e} 2$ & $8.76 \mathrm{e} 4$ \\
\hline
\end{tabular}

Table 6 Material properties of a non-symmetric 3-layered cantilever beam.

The beam has been analyzed with meshes of 5, 10 and 20 EEBZ2 elements. Results are denoted as EEBZ2-5, EEBZ2-10 and EEBZ2-20 respectively.

For comparison purposes this problem was also analyzed with a mesh of 50 two-noded TBT elements, which results are denoted as TBT-50.

The problem was also solved with a structured mesh of 18048 four-noded plane stress quadrilateral elements, which is considered as the reference solution of the problem. 
Figure 10 shows the deflection values along the beam length. The results of the EEBZ2-5 mesh agree well with the plane stress solution. The TBT results are far from the correct ones.

Figures 11 to 13 show respectively the thickness distribution for the axial displacements and the axial and tangential stresses at a beam section located at $x=L / 4$ from the clamped end.

Figures 14 to 16 show respectively the thickness distribution of the axial displacements, and the axial and tangential stresses at a beam section for $x=3 L / 4$.

Excellent results were obtained with the EEBZ2-10 mesh.

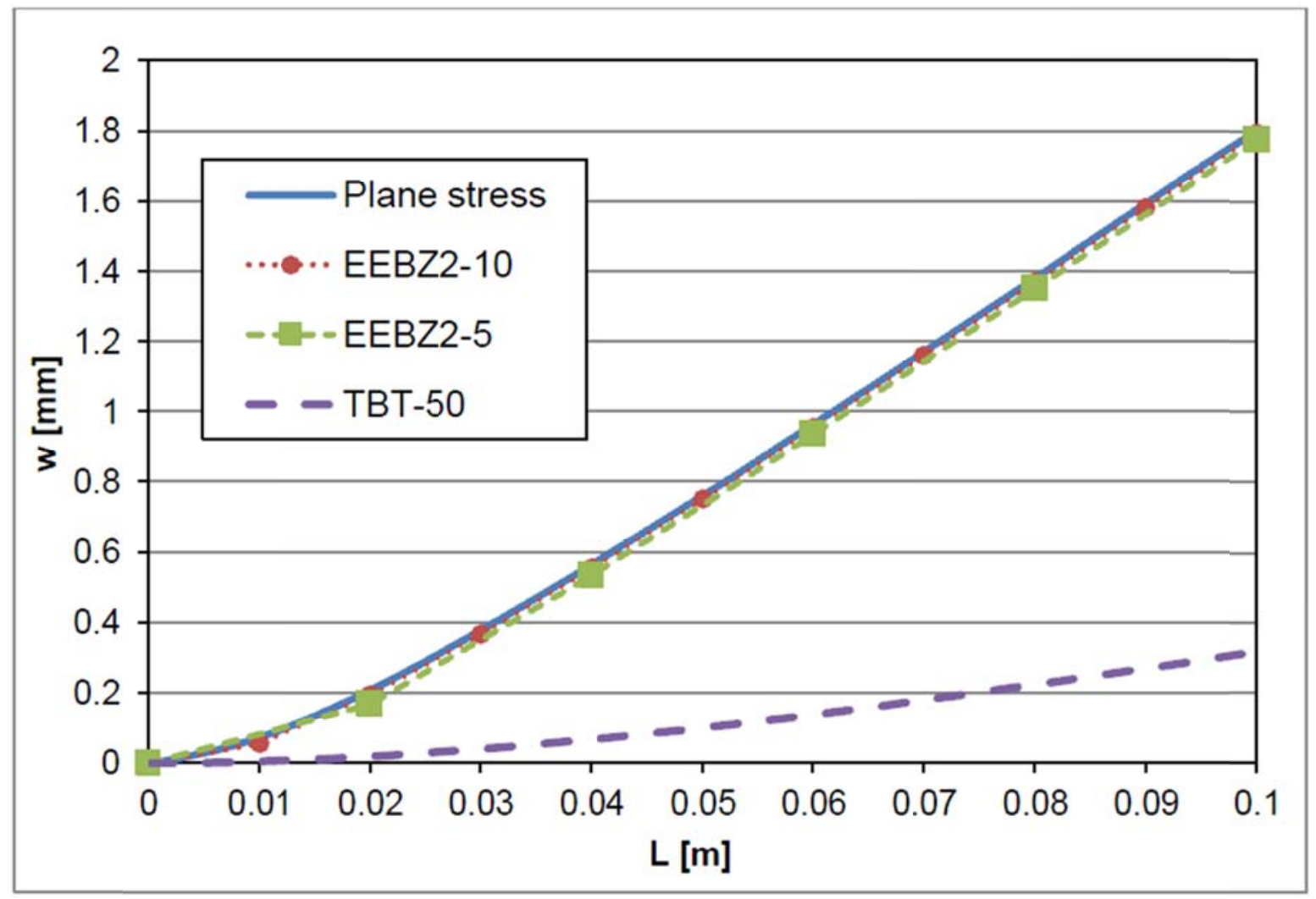

Figure 10 Non-symmetric 3-layered cantilever beam under end-point load. Distribution of the vertical deflection $w$ for different theories and meshes. 


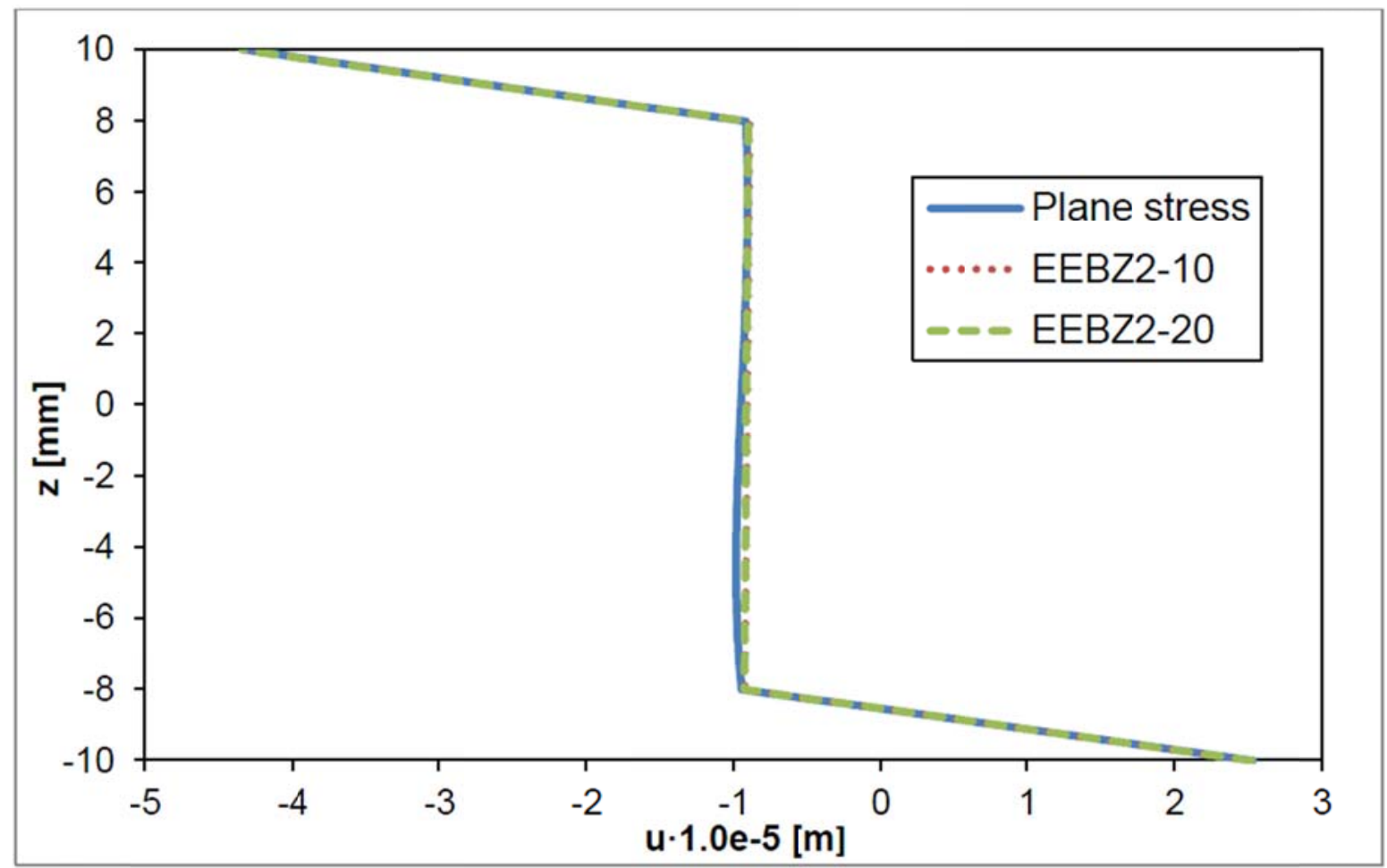

Figure 11 Non-symmetric 3-layered cantilever beam under end-point load. Thickness distribution of the axial displacement $\mathrm{u}$ at $\mathrm{x}=\mathrm{L} / 4$.

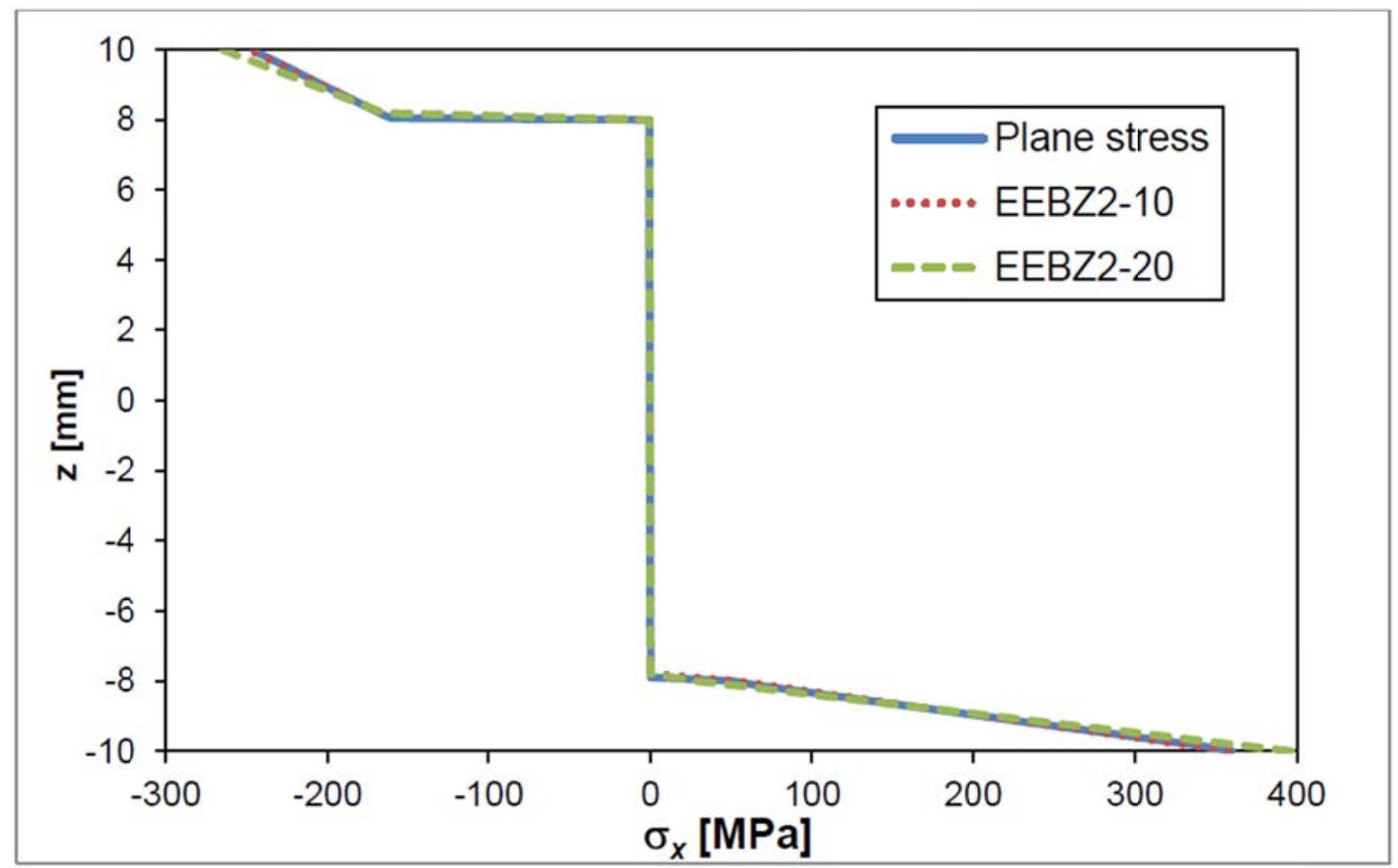

Figure 12 Non-symmetric 3-layered cantilever beam under end-point load. Thickness distribution of the axial stress $\sigma_{x}$ at $x=L / 4$. 


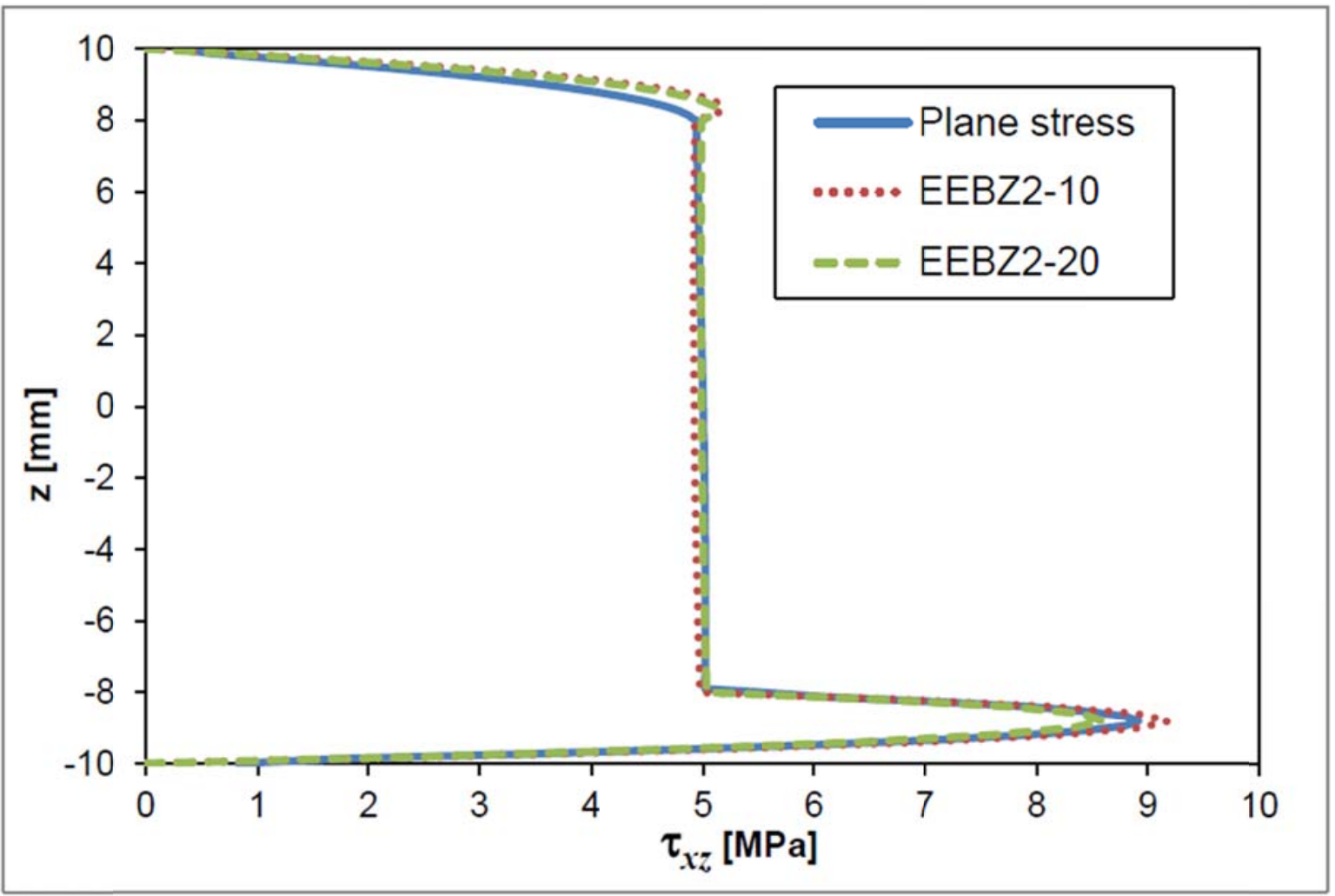

Figure 13 Non-symmetric 3-layered cantilever beam under end-point load. Thickness distribution of the tangential stress $\tau_{x z}$ at $x=L / 4$.

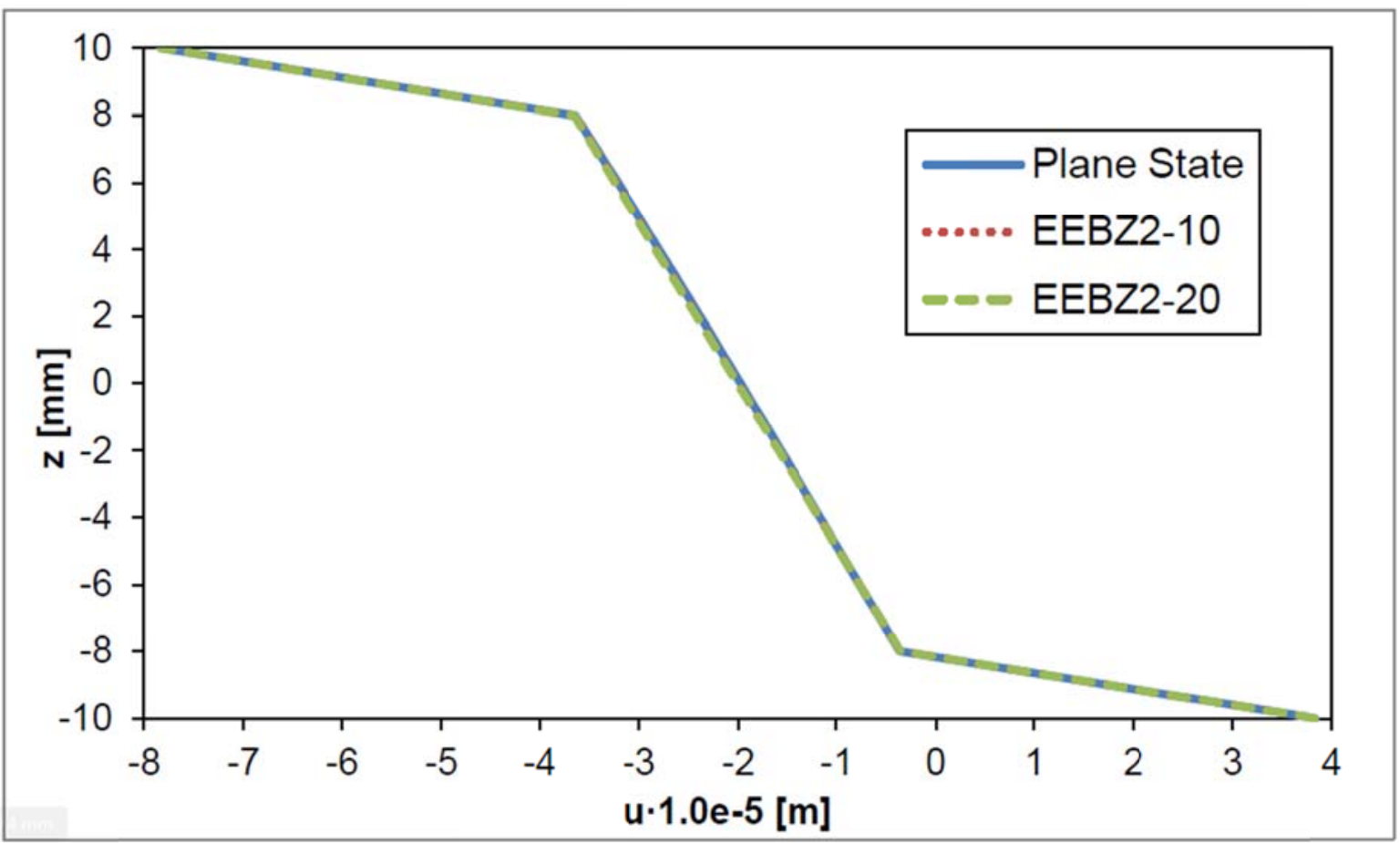

Figure 14 Non-symmetric 3-layered cantilever beam under end-point load. Thickness distribution of the axial displacement $\mathrm{u}$ at $x=3 \mathrm{~L} / 4$. 


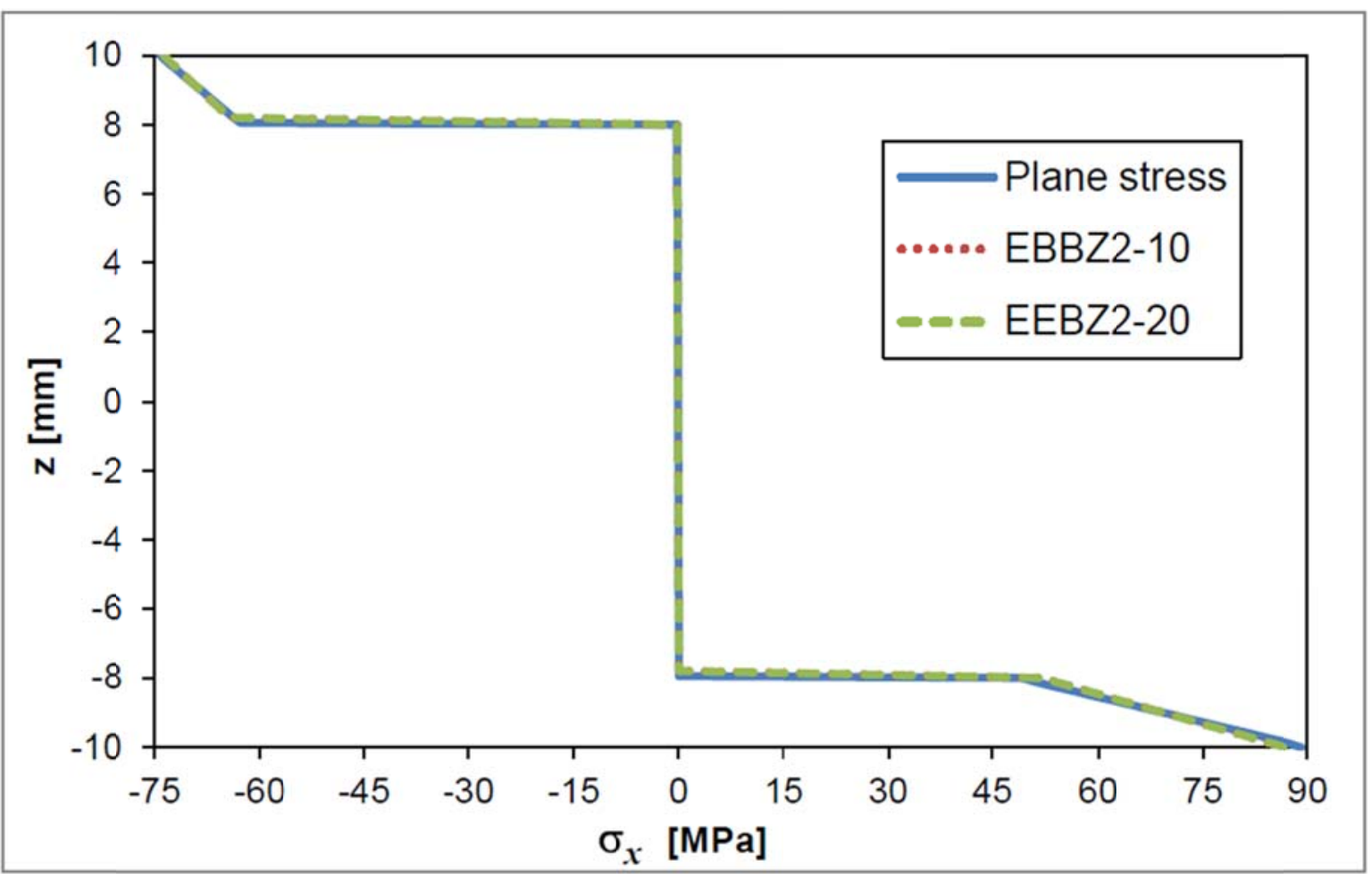

Figure 15 Non-symmetric 3-layered cantilever beam under end-point load. Thickness distribution of the axial stress $\sigma_{x}$ at $x=3 L / 4$.

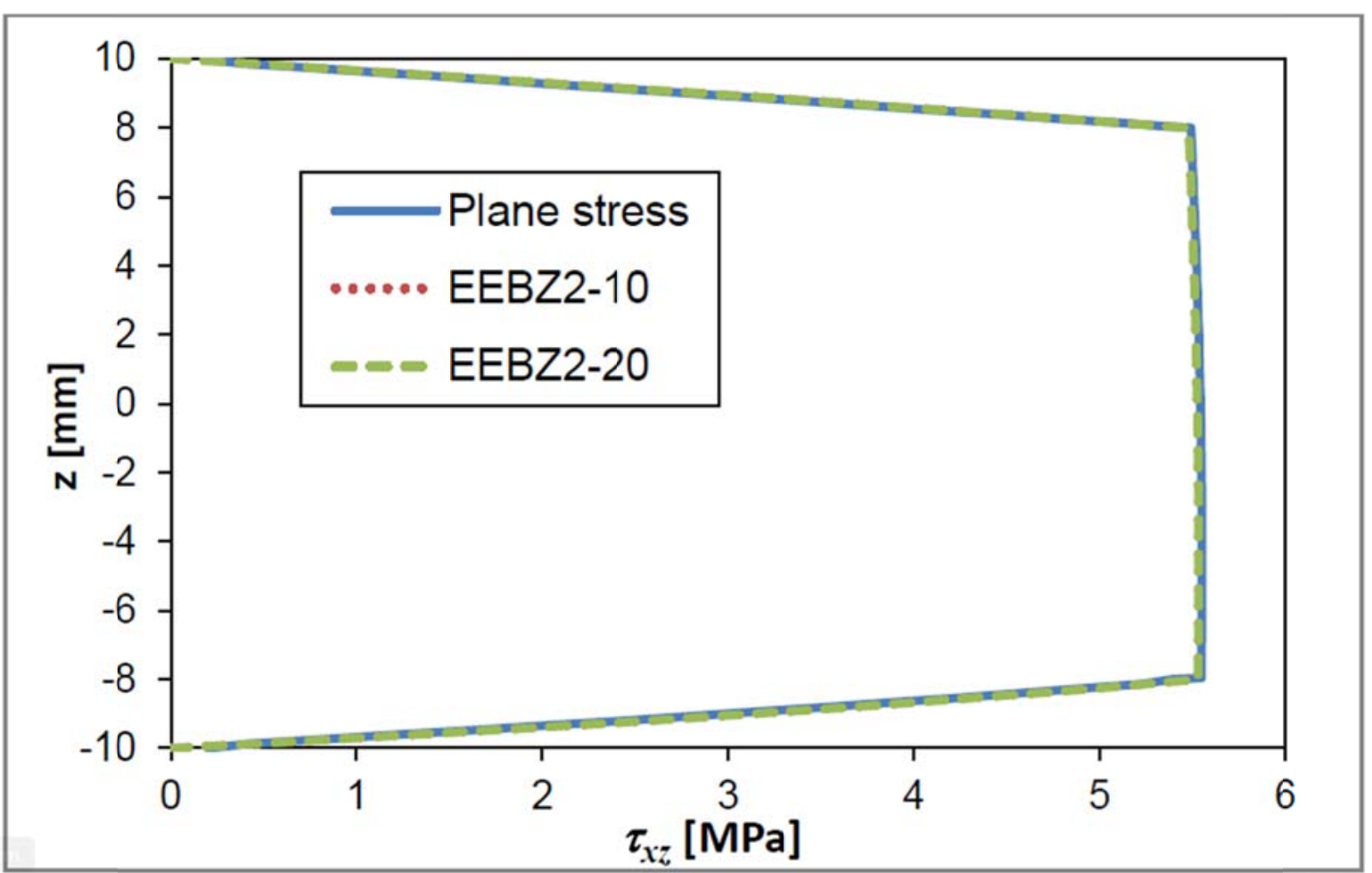

Figure 16 Non-symmetric 3-layered cantilever beam under end-point load. Thickness distribution of the axial stress $\tau_{x z}$ at $x=3 L / 4$. 


\section{6-3) Non-symmetric three-layered simple support thick beam under uniform load}

In this example a three-layered simple supported thick rectangular beam under a uniform distributed load is analyzed (Figure 17). The material properties are presented in Table 4. The span-to-thickness ratio is $\lambda=5$.

The three layers have the same thickness but their mechanical properties are very different. The central layer has very low values of the Young and shear moduli.
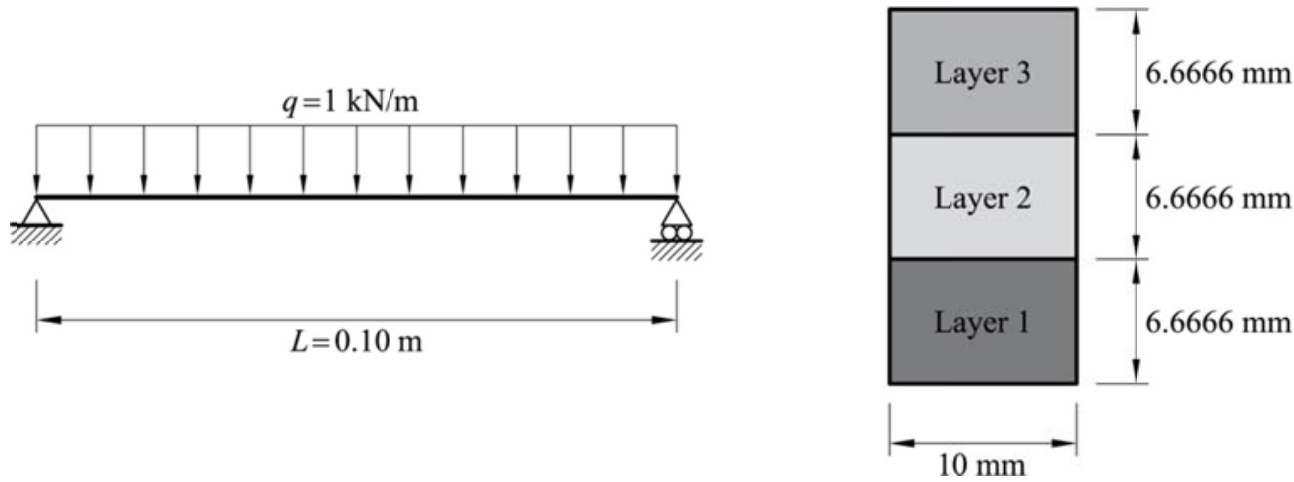

Figure 17 Three-layered laminated thick cantilever beam under end point load.

\begin{tabular}{|c|c|c|c|}
\cline { 2 - 4 } \multicolumn{1}{c|}{} & Layer 1 & Layer 2 & Layer 3 \\
\hline $\mathrm{h}[\mathrm{mm}]$ & 6.6666 & 6.6666 & 6.6666 \\
\hline $\mathrm{E}[\mathrm{MPa}]$ & $2.19 \mathrm{e} 5$ & $5.30 \mathrm{e} 2$ & $7.30 \mathrm{e} 5$ \\
\hline $\mathrm{G}[\mathrm{MPa}]$ & $0.876 \mathrm{e} 5$ & $2.12 \mathrm{e} 2$ & $2.92 \mathrm{e} 5$ \\
\hline
\end{tabular}

Table 7 Material properties of a non-symmetric 3-layered simply supportted beam.

The beam was analyzed with meshes of 10 and 20 EEBZ2 elements, which results are denoted as EEBZ2-10 and EEBZ2-20 respectively. The results are compared to those obtained with a mesh of 50 two-noded TBT elements and with a structured mesh of 8000 four-noded plane stress quadrilateral elements.

Figure 18 shows the deflection values along the beam length. The TBT results are significantly stiffer.

Figures 19 to 21 show respectively the thickness distribution of the axial displacements, and the axial and tangential stresses at the beam section for $x=L / 4$. Very good agreement of the EEBZ2-10 mesh with the plane stress results is again obtained. 


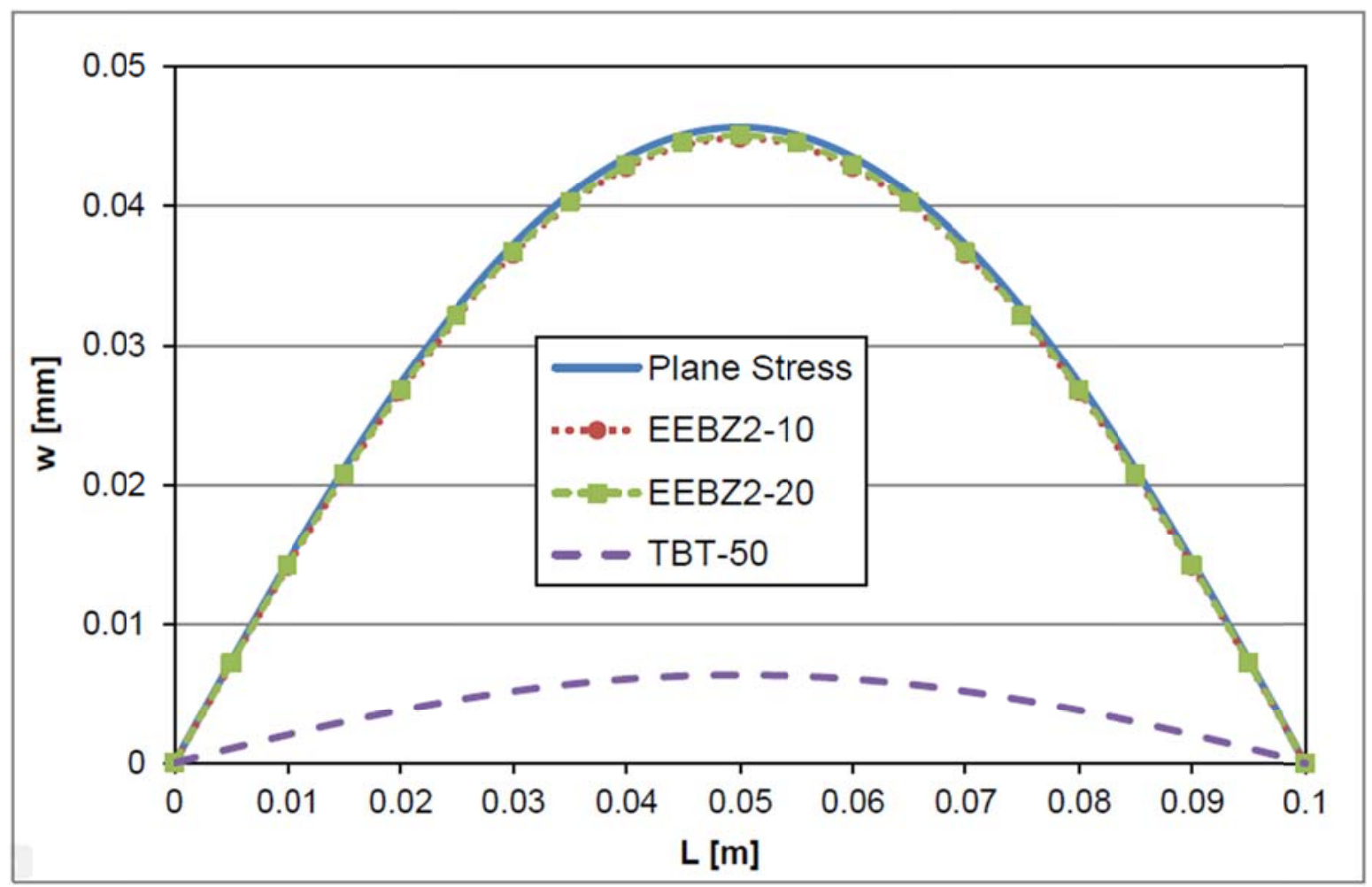

Figure 18 Non-symmetric 3-layered simple supported beam under uniform distributed load. Distribution of the vertical deflection $w$ for different theories and meshes.

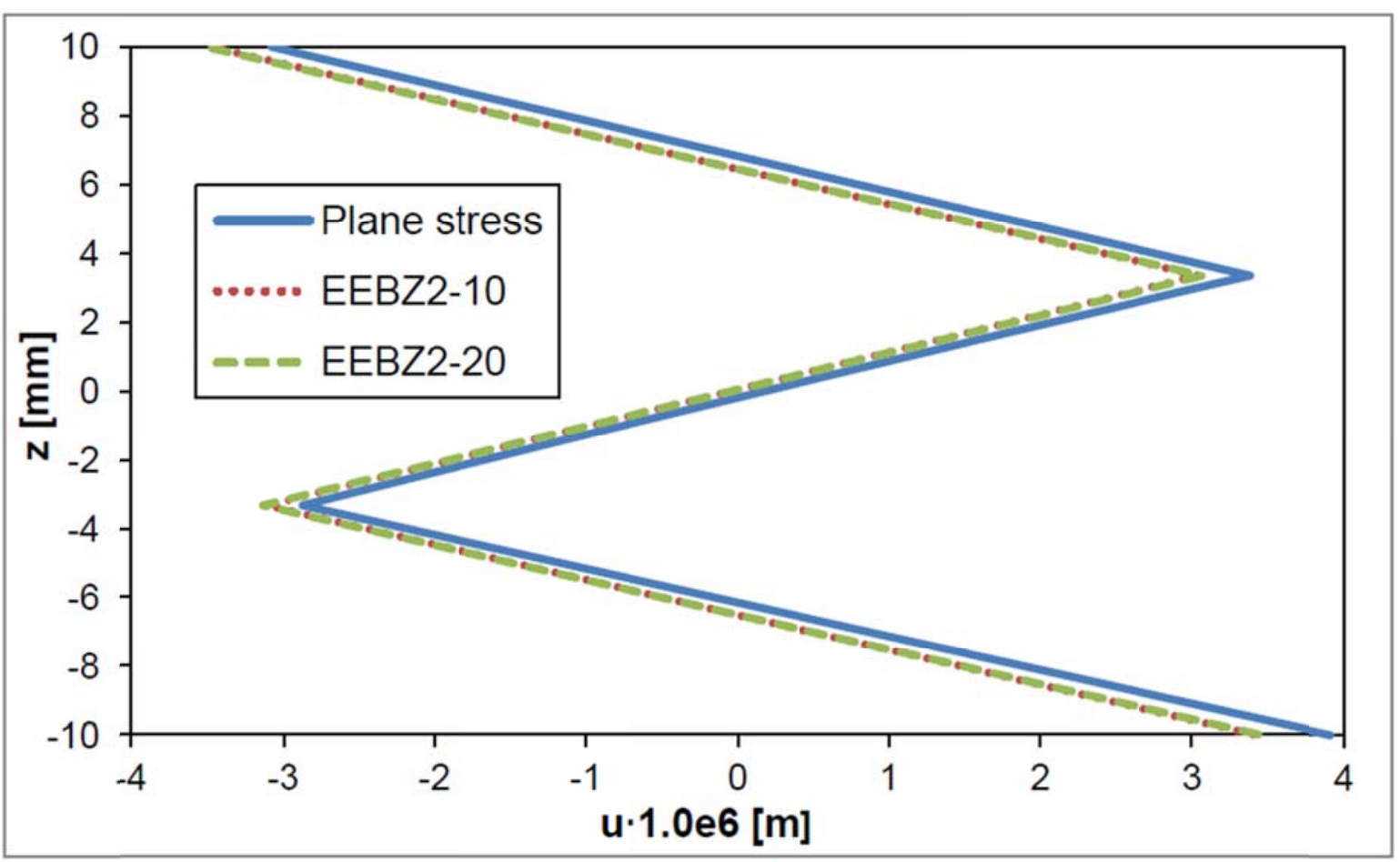

Figure 19 Non-symmetric 3-layered simple supported beam under uniform distributed load. Thickness distribution of the axial displacement $u$ at $x=L / 4$. 


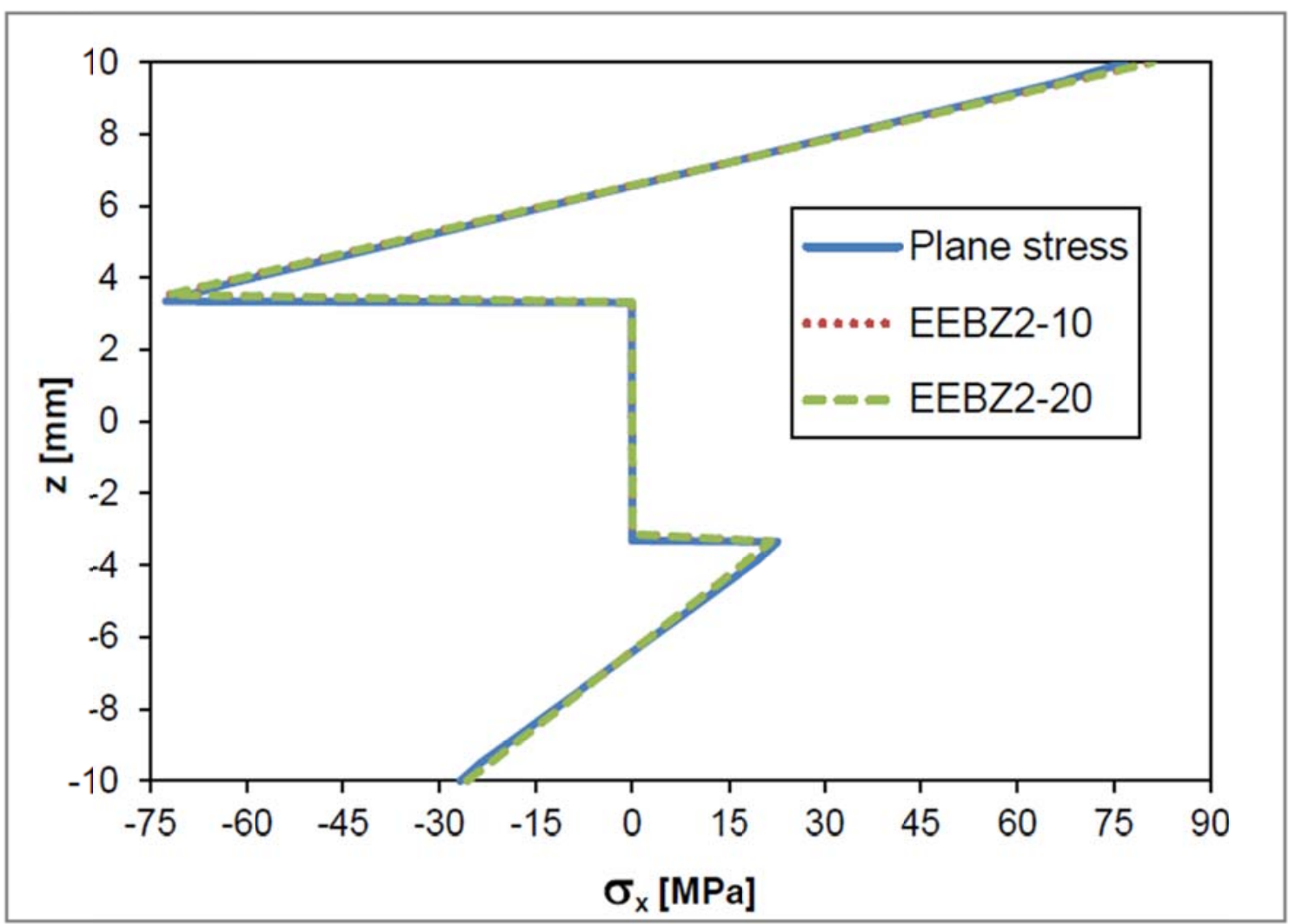

Figure 20 Non-symmetric 3-layered simple supported beam under uniform distributed load. Thickness distribution of the axial stress $\sigma_{x}$ at $x=L / 4$.

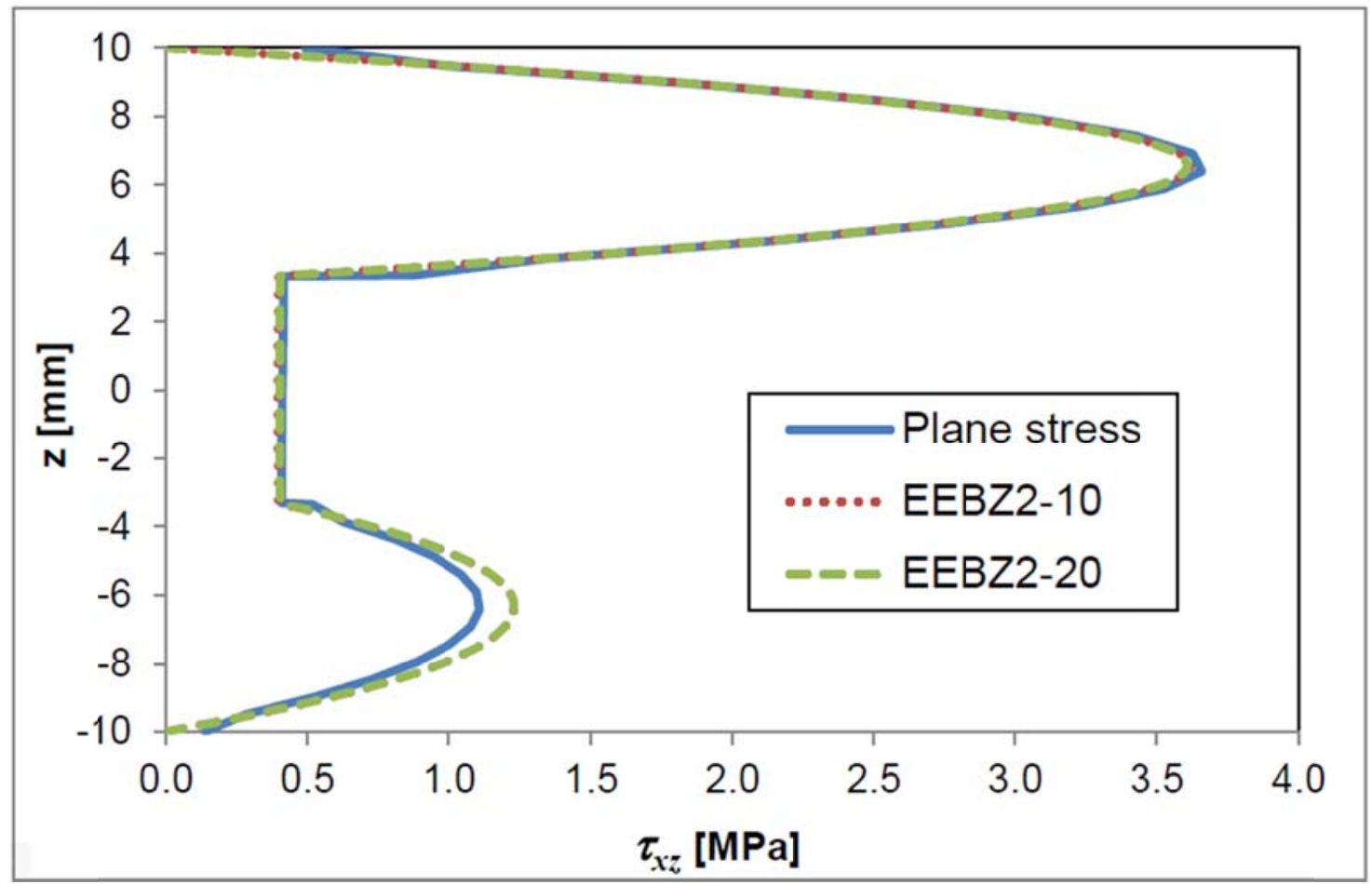

Figure 21 Non-symmetric 3-layered simple supported beam under uniform distributed load. Thickness distribution of the axial stress $\tau_{x z}$ at $x=L / 4$. 


\section{6-4) Non-symmetric ten-layered clamped slender beam under uniform load}

In this example a ten-layered clamped supported slender rectangular beam under uniform distributed load is analyzed (Figure 22). A non-symmetric stacking configuration for the laminate is presented in Table 5. The span-to-thickness ratio is $\lambda=20$.
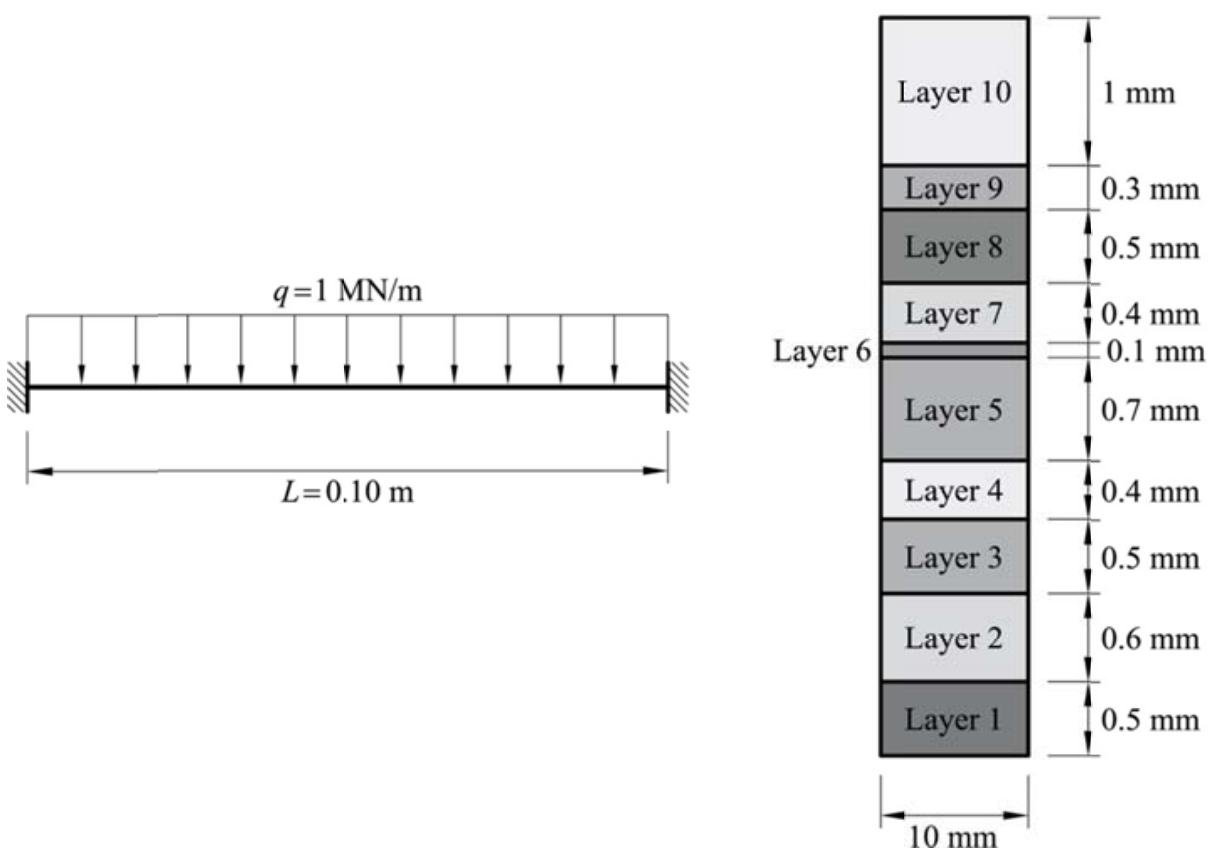

Figure 22 Ten-layered laminated clamped slender beam under uniform load.

\begin{tabular}{|c|c|c|c|}
\cline { 2 - 4 } \multicolumn{1}{c|}{} & $\mathrm{h}[\mathrm{mm}]$ & $\mathrm{E}[\mathrm{MPa}]$ & $\mathrm{G}[\mathrm{MPa}]$ \\
\hline Layer 1 & 0.5 & $5.30 \mathrm{e} 5$ & $2.12 \mathrm{e} 5$ \\
\hline Layer 2 & 0.6 & $2.19 \mathrm{e} 5$ & $0.876 \mathrm{e} 5$ \\
\hline Layer 3 & 0.5 & $0.82 \mathrm{e} 5$ & $0.328 \mathrm{e} 5$ \\
\hline Layer 4 & 0.4 & $7.30 \mathrm{e} 2$ & $2.92 \mathrm{e} 2$ \\
\hline Layer 5 & 0.7 & $5.30 \mathrm{e} 5$ & $2.12 \mathrm{e} 5$ \\
\hline Layer 6 & 0.1 & $7.30 \mathrm{e} 2$ & $2.92 \mathrm{e} 2$ \\
\hline Layer 7 & 0.4 & $7.30 \mathrm{e} 5$ & $2.92 \mathrm{e} 5$ \\
\hline Layer 8 & 0.5 & $0.82 \mathrm{e} 5$ & $0.328 \mathrm{e} 5$ \\
\hline Layer 9 & 0.3 & $2.19 \mathrm{e} 5$ & $0.876 \mathrm{e} 5$ \\
\hline Layer 10 & 1.0 & $7.30 \mathrm{e} 5$ & $2.92 \mathrm{e} 5$ \\
\hline
\end{tabular}

Table 8 Material properties of a non-symmetric 10-layered clamped supported beam.

The beam was analyzed with meshes of 10 and 20 EEBZ2 elements. Similarly to the previous examples the results obtained with a mesh a 50 two-noded TBT elements and with a structured mesh of 40000 four-noded plane stress quadrilaterals are also presented.

Figure 23 shows the deflection values along the beam length. The error for the EEBZ2-10 mesh versus the plane stress results is around of $6 \%$. Similarly to the previous examples the TBT results are far from the correct ones. 
Figures 24 to 26 show respectively the thickness distribution of the axial displacement, and the axial and tangential stresses at the beam section for $x=L / 4$. Excellent results are again obtained with the EEBZ2-10 mesh.

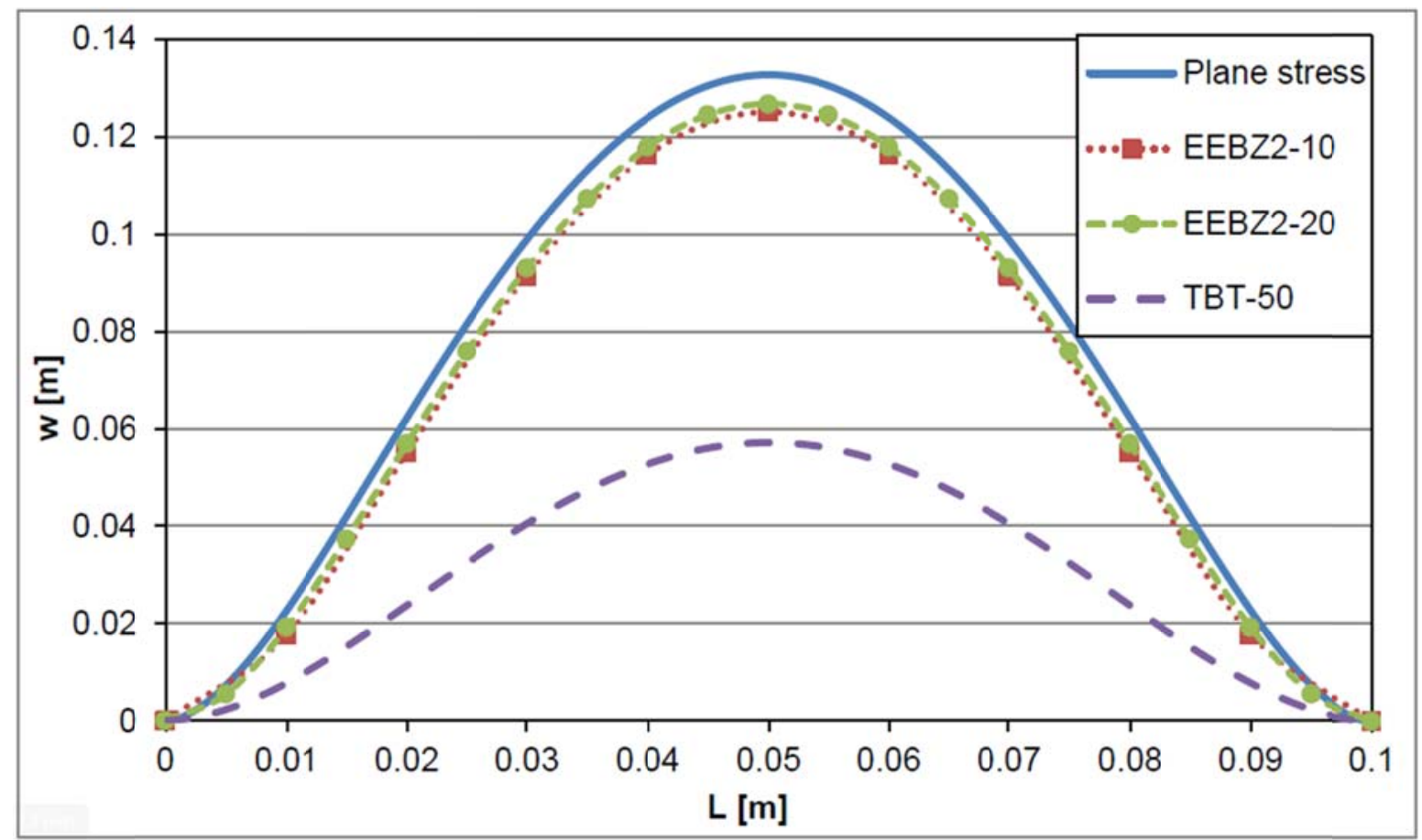

Figure 23 Non-symmetric 10-layered clamped supported beam under uniform distributed load. Distribution of the vertical deflection $w$ for different theories and meshes.

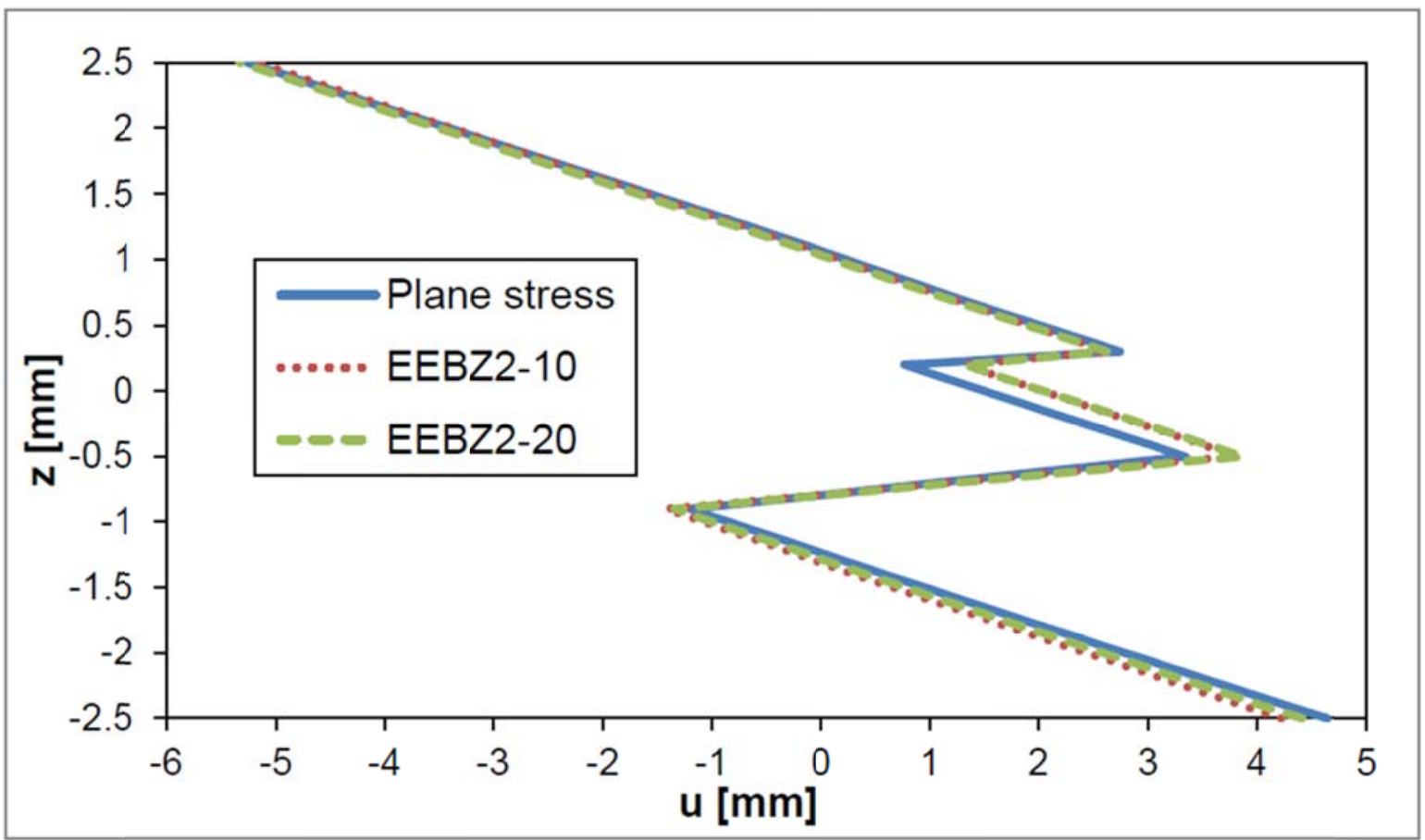

Figure 24 Non-symmetric 10-layered clamped supported beam under uniform distributed load. Thickness distribution of the axial displacement $u$ at $x=L / 4$. 


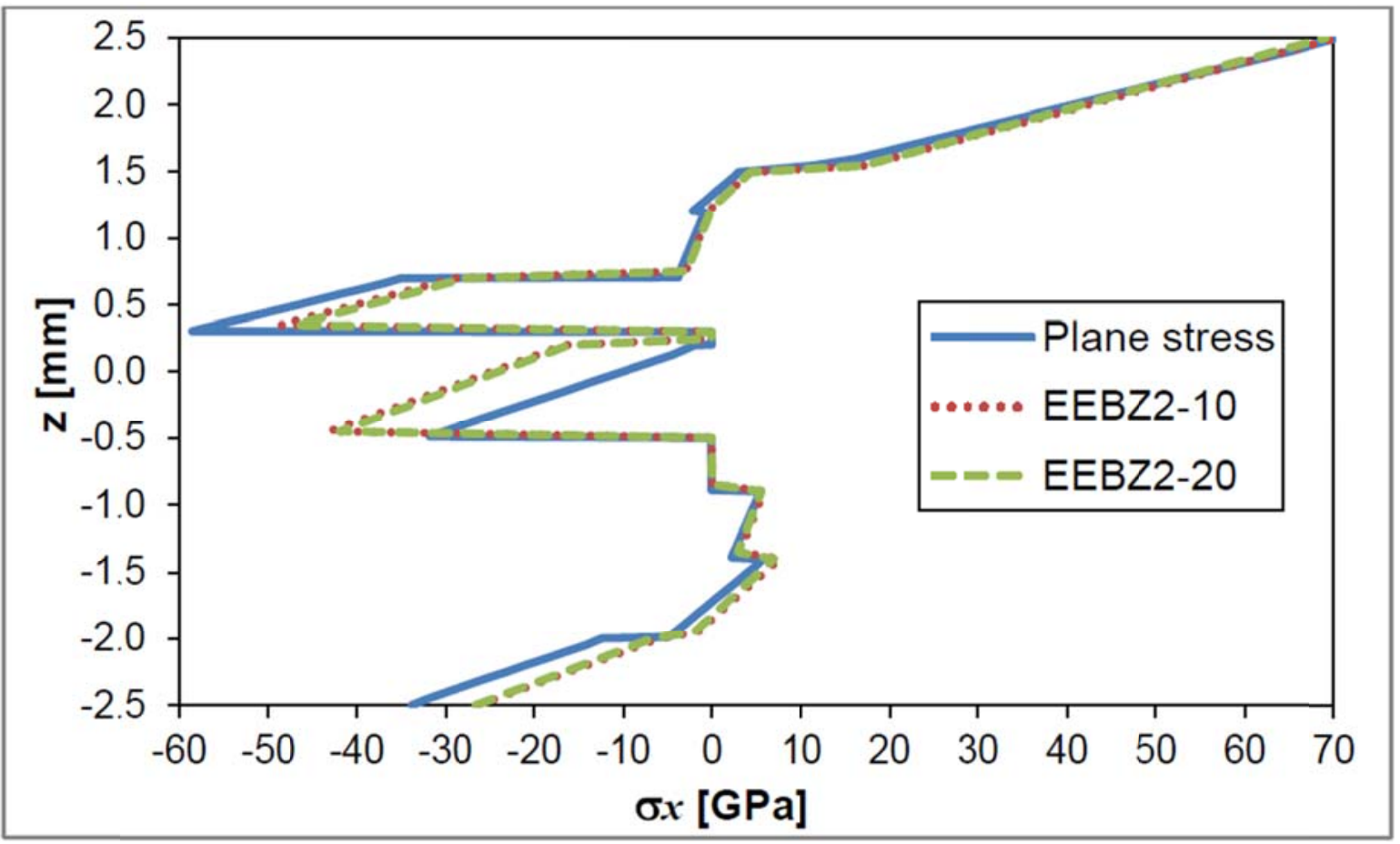

Figure 25 Non-symmetric 10-layered clamped supported beam under uniform distributed load. Thickness distribution of the axial stress $\sigma_{x}$ at $x=L / 4$.

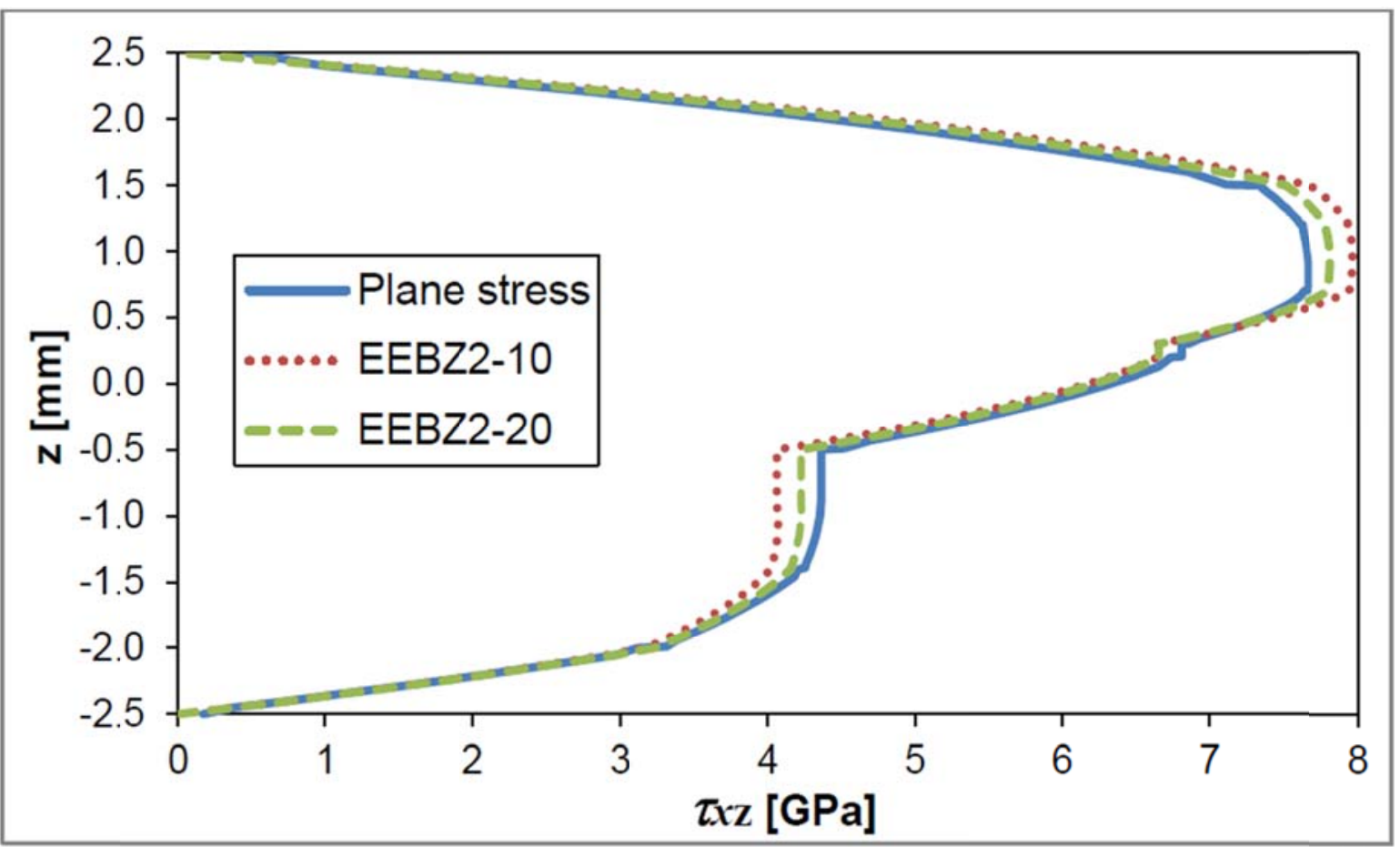

Figure 26 Non-symmetric 10-layered clamped supported beam under uniform distributed load. Thickness distribution of the axial stress $\tau_{x y}$ at $x=L / 4$. 


\section{6-5) Modeling of delamination with the EEBZ2 element}

In this example a delamination situation in a laminated thick cantilever rectangular beam under an end point load is analyzed (Figure 27). The material properties are non-symmetric and are presented in Table 6 . The span-to-thickness ratio is $\lambda=5$. Delamination between the upper and core layers has been modeled by introducing a zero thickness layer in the interface between these two layers (Figure 28). This method is an extension of the approach suggested in $[20,22]$. The degree of delamination is controlled by the parameter $\beta_{d}$ defined in Section 2-1, which ranges between 0 and 1 .
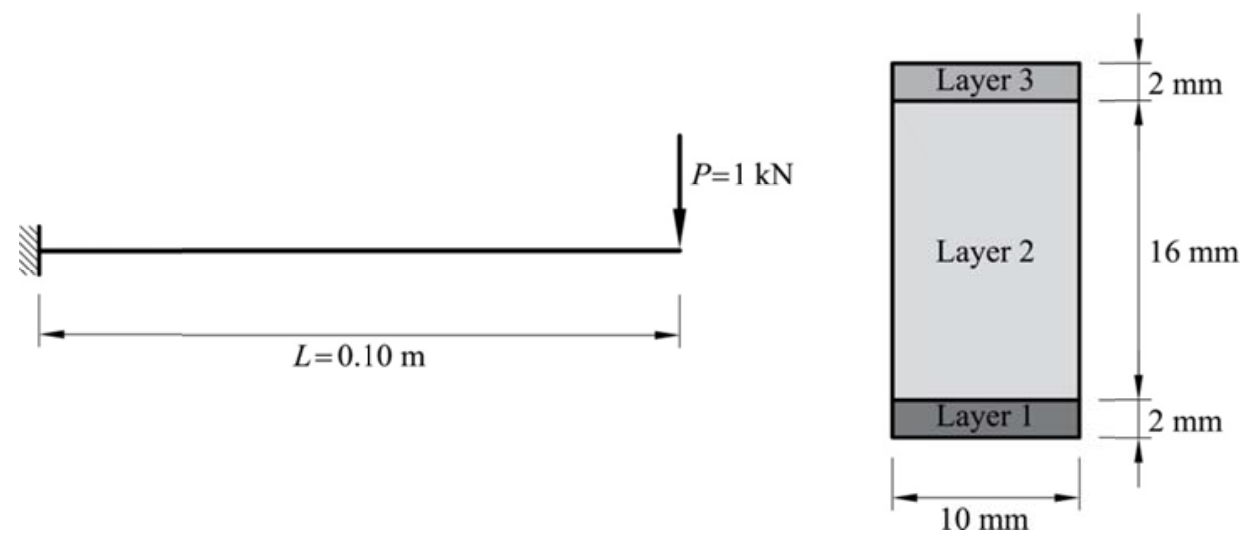

Figure 27 Three-layered laminated thick cantilever beam under end point load.

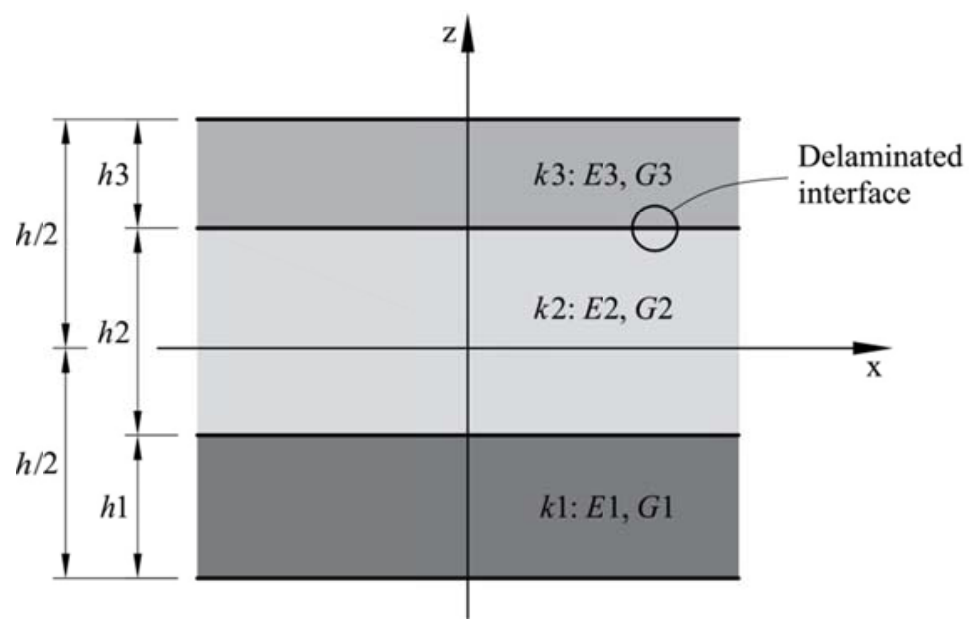

Figure 28 Modeling of a zero thickness interface layer for the delamination study in a 3-layered thick cantilever beam $(\lambda=5)$ under end point load.

\begin{tabular}{|c|c|c|c|}
\cline { 2 - 4 } \multicolumn{1}{c|}{} & Layer 1 & Layer 2 & Layer 3 \\
\hline $\mathrm{h}[\mathrm{mm}]$ & 2 & 16 & 2 \\
\hline $\mathrm{E}[\mathrm{MPa}]$ & $7.30 \mathrm{e} 5$ & $7.30 \mathrm{e} 2$ & $2.19 \mathrm{e} 5$ \\
\hline $\mathrm{G}[\mathrm{MPa}]$ & $2.92 \mathrm{e} 5$ & $2.20 \mathrm{e} 2$ & $8.76 \mathrm{e} 4$ \\
\hline
\end{tabular}

Table 9 Material properties of non-symmetric 3-layered cantilever beam. 
The same beam is analyzed using meshes of 18240 four-noded plane stress rectangles. The delaminated interface is modeled by a very thin row of elements $(h=0.01 \mathrm{~mm})$. The degree of delamination is controlled by the shear modulus for this thin row of elements. Only two situations are considered, an initial state without delamination $\left(\mathrm{G}=8.76 \mathrm{e}^{4} \mathrm{MPa}\right)$ and a final state with full delamination $\left(\mathrm{G}=8.76 \mathrm{e}^{-7} \mathrm{MPa}\right)$.

Figure 29 shows results for the end deflection in terms of the sectional delamination parameter $\beta_{d}$ for the EEBZ2-10 and EEBZ2-20 meshes. The end deflection values are normalized with the deflection in the initial non-delaminated situation, i.e. for $\beta_{d}=0$. The results of the plane stress analysis for a normalized value of the end deflection and for a full delamination situation are also presented.

It can be appreciated that the error of the EEBZ2-10 mesh versus the plane stress result is less than $2 \%$.

Figures 30 to 32 show respectively the thickness distribution of the axial displacement, the axial stress and the tangential stresses at a beam section for $x=L / 2$. Results are in a good agreement with the plane stress results.

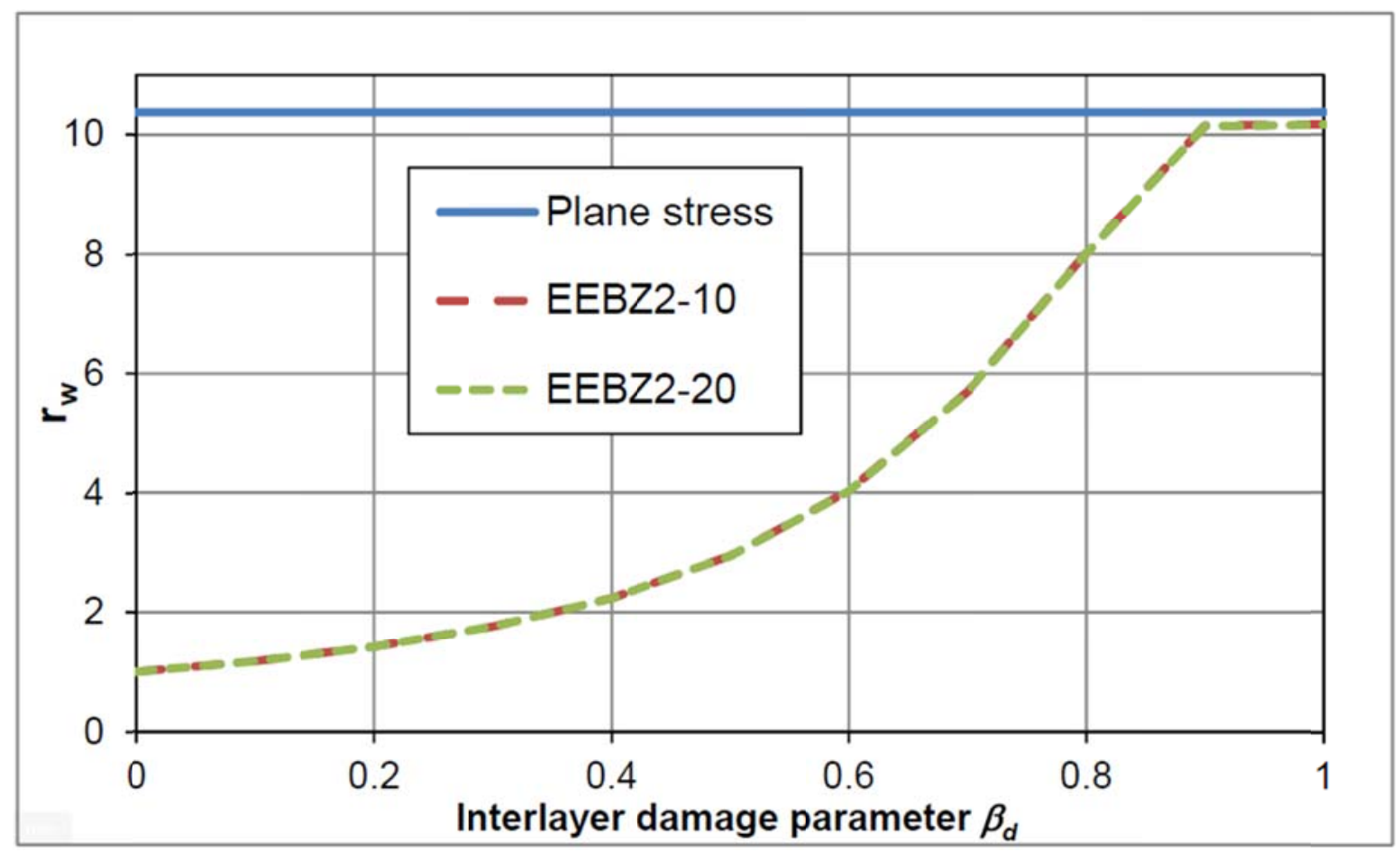

Figure 29 Delamination study in 3-layered cantilever beam under end point load. Evolution of the normalized end deflection value with the intefase delamination parameter $\beta_{d}$ for the EEBZ2-10 and EEBZ2-20 meshes and the plane stress solution. 


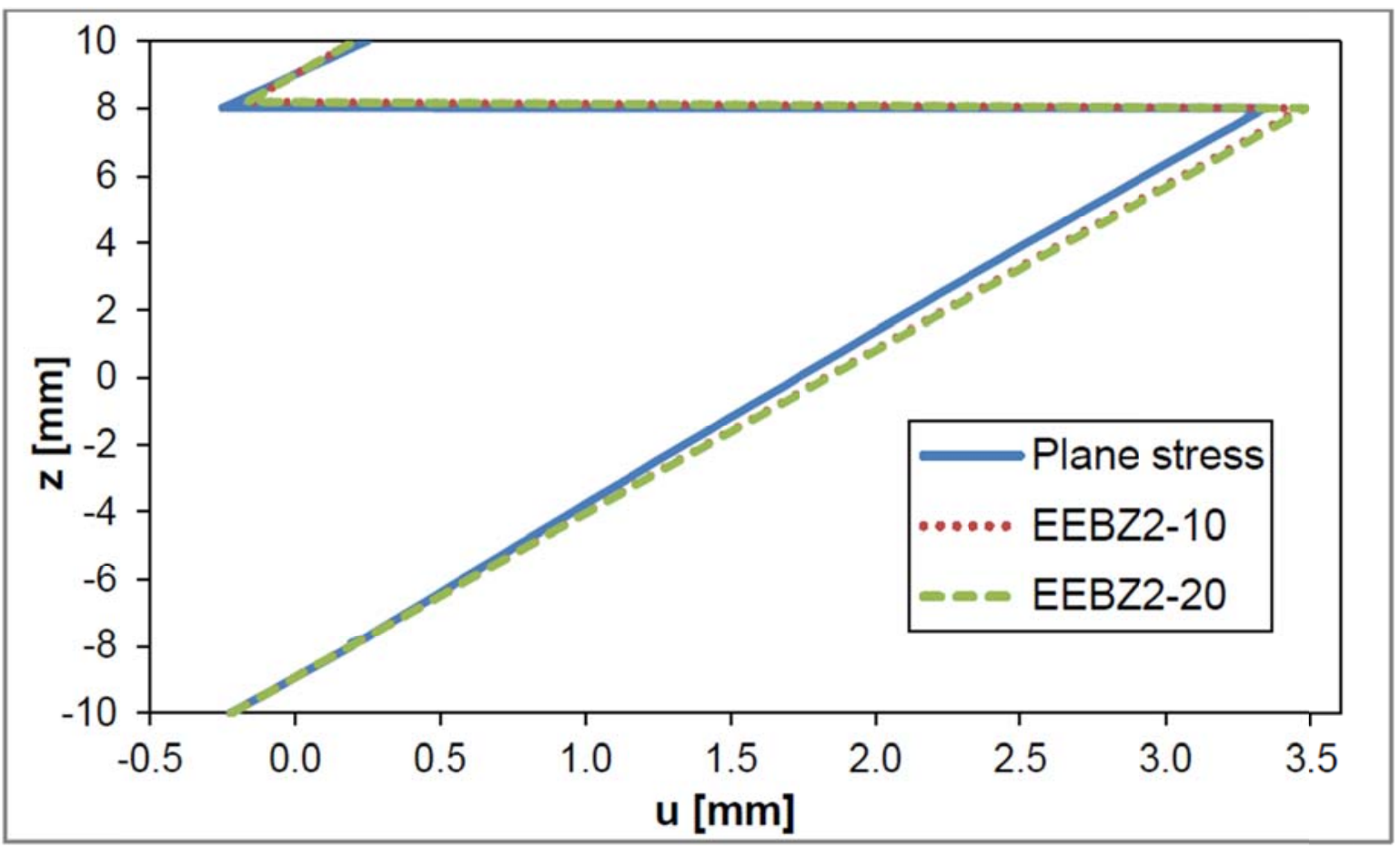

Figure 30 Delamination study in 3-layered cantilever beam under end point load. Thickness distribution of the axial displacement $u$ at $x=L / 2$.

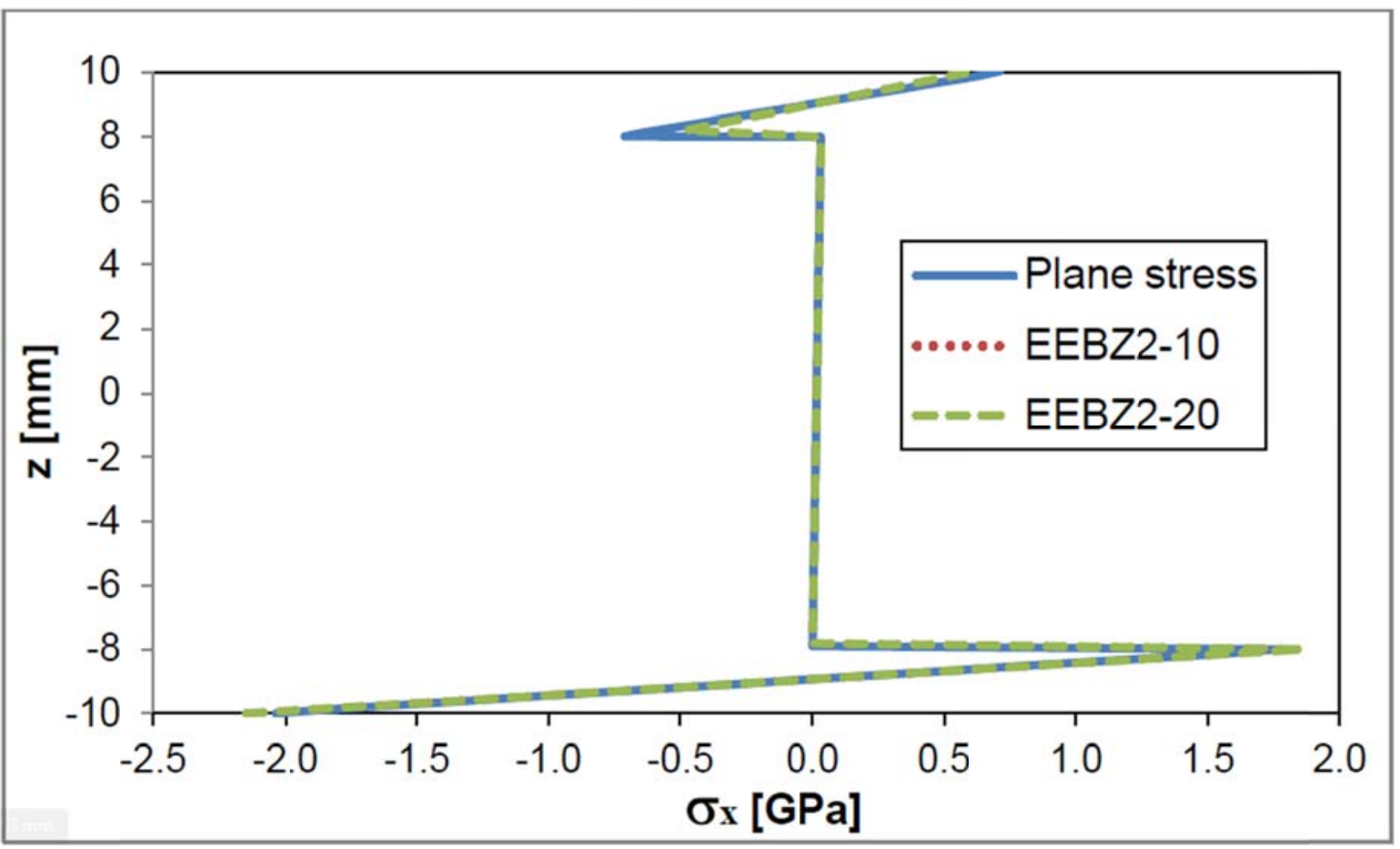

Figure 31 Delamination study in 3-layered cantilever beam under end point load. Thickness distribution of the axial stress $\sigma_{x}$ at $x=L / 2$. 


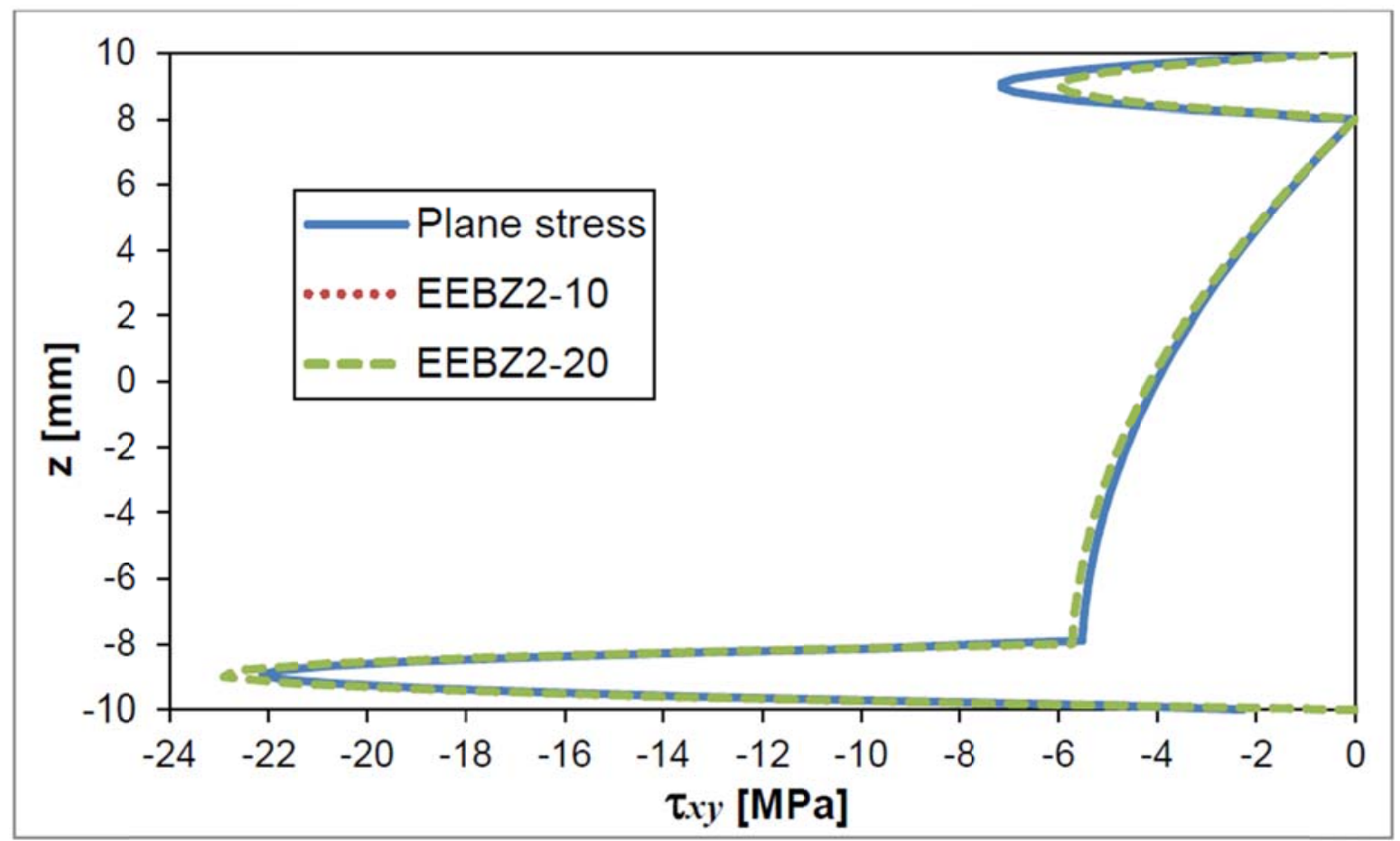

Figure 32 Delamination study in 3-layered cantilever beam under end point load. Thickness distribution of the axial stress $\tau_{x z}$ at $x=L / 2$.

\section{7-) Conclusions}

We have present a new 2-noded zigzag beam element (termed EEBZ2) for the analysis of composite laminate and sandwich beams which is able to take into account distortion effects due to shear elastic strains. The element can also predict delamination situations. The kinematic assumptions are based in the RZT [18] and the classical EBT. The element has four degrees of freedom per node (the axial displacement $u_{0}$, the deflection $w_{0}$, the deflection slope $d w_{0} / d x$ and the shear angle $\psi$ ). A $C^{1}$ cubic Hermite interpolation is used for the deflection and a $C^{0}$ linear interpolation for the other variables.

The apparent theoretical difficulties of the $C^{1}$ deflection interpolation to represent correctly shear strains in clamped support is overcome by considering that the shear strains resulting from the formulation are only a part of the total shear strains. We have shown that this part of the total shear strains is enough to yield an accurate approximation for the deflection along the beam and for the distribution of the axial displacements and the axial stresses across the thickness. An enhanced distribution of the total shear stress is obtained in a post-processing step by integrating the equilibrium equations using the computed values of the axial stress.

The advantages of the EEBZ2 beam element versus those based in the classical TBT are several. On one hand, the EEBZ2 element is free of shear locking. On the other hand, the stiffness matrix can be explicitly calculated in closed form using exact integration.

Delamination effects are modeled by introducing an additional zigzag function corresponding to the kinematics of a zero thickness delaminate layer. This approach avoids the need to choose suitable small values for thickness and the shear modulus in a very thin interface layer for simulating the delamination as required in [21]. 


\section{Acknowledgements}

Authors would like to acknowledge to Prof. Sergio Oller for his valuable comments and suggestions. 


\section{References}

[1] Timoshenko S. On the correction for shear of differential equations for transverse vibration of prismatic bars. Philosophical Magazine Series 6 1921: 41(245):744-746.

[2] Liu D, Li X. An overall view of laminate theories based on displacement hypothesis. Journal of Composite Materials 1996; 30(14):1539-1561.

[3] Reddy JN. A refined nonlinear theory of plates with transverse shear deformation. Computers and structures 1987; 20( 9-10):881-896.

[4] Reddy JN. A generalization of two-dimensional theories of laminated composite plates. Communications in Applied Numerical Methods 1987; 3(3):173-180.

[5] Reddy JN. On refined computational models of composite laminates. International Journal for Numerical Methods in Engineering 1989; 27(2):361-382.

[6] Di Sciuva M. Bending, vibration and buckling of simply supported thick multilayered orthotropic plates: An evaluation of a new displacement model. Journal of Sound and Vibration 1986; 105(3):425-442.

[7] Murakami H. Laminated composite plate theory with improved in-plane. Journal of Applied Mechanics 1986; 53(3):661-666.

[8] Aitharaju VR, Averill RC. C0 Zig-Zag Finite Element for Analysis of Laminated Composite Beams. Journal of Engineering Mechanics 1999; 125(3):323-330.

[9] Lu X, Liu D. An interlaminar shear stress continuity theory for both thin and thick composite laminate. Journal of Applied Mechanics 1992; 59( 3):502-509.

[10] Di Sciuva M. An improved shear-deformation theory for moderately thick. Journal of Applied Mechanics 1987; 54(3):589-596.

[11] Toledano A, Murakam, H. A higher-order laminate plate theory with. International Journal of Solids and Structures 1987; 23(1):111-131.

[12] Cho M, Parmerter R. Efficient higher order composite plate theory for general lamination configurations, AIAA Journal 1993, 31(7):1299-1306.

[13] Kapuria S, Dumir P, Ahmed A, Alam N. Finite element model of efficient zig-zag theory for static analysis of hybrid piezoelectric beams. Computational Mechanics 2004; 34(6): 475 486.

[14] Di Sciuva M. Multilayered anisotropic plate model with continuous interlaminar stresses Composite Structures 1992; 22(3):149-167.

[15] Alam NM, Upadhyay N.Kr. Finite Element Analysis of Laminated Composite Beams for Zigzag Theory using MATLAB. International Journal of Mechanics and Solids 2010; 5(1):1-14.

[16] Averill RC. Static and dynamic response of moderately thick laminated beams with damage. Composites Engineering 1994, 4(4):381-395. 
[17] Averill R, Yip Yuen Cheong. Development of simple, robust finite elements based on refined theories for thick laminated beams. Computers and Structures 1996; 59(3):529-546.

[18] Tessler A, Di Sciuva M, Gherlone M. A refined zigzag beam theory for composite and sandwich beams. Journal of Composite Materials 2009; 43(9)1051-1081.

[19] Tessler A, Di Sciuva M, Gherlone M. A consistent refinement of first-order shear deformation theory for laminated composite and sandwich plates using improved zigzag kinematics. Journal of Mechanics of Materials and Structures 2010; 5(2):341-367.

[20] Gherlone M, Tessler A, Di Sciuva, M. C0 beam element based on the refined zigzag theory for multilayered composite and sandwich laminates. Composite Structures 2011; 93(11):28822894.

[21] Oñate E, Eijo A, Oller S. Simple and accurate two-noded beam element for composite laminate beam using a refined zigzag theory. Computer Methods in Applied Mechanics and Engineering 2012; 213-216(1):362-382.

[22] Eijo A, Oñate E, Oller S. A numerical model of delamination in composite laminated beams using the LRZ beam element based on the refined zigzag theory. Composite Structures 2013; 104(1):270-280.

[23] Oñate, E. Structural analysis with the finite element method. Linear Static. Volumen 2. Beams, Plates and Shells, Springer, 2013.

[24] Icardi U, Zardo G. C0 plate element for delamination damage analysis, based on a zig-zag model and strain energy updating. International Journal of Impact Engineering 2005;31(5):579606.

[25] Cho M, Kim JS. Higher-order zig-zag theory of laminated composites with multiple delaminations. Journal of Applied Mechanics 2001;68(6):869-77.

[26] Kim JS, Cho M. Efficient higher-order shell theory for laminated composites with multiple delaminations. AIAA J 2003;41(5):941-50.

[27] Oh J, Cho M, Kim JS. Dynamic analysis of composite plate with multipledelaminations based on higher-order zigzag theory International Journal of Solids and Structures $2005 ; 42: 6122-40$.

[28] Di Sciuva M, Gherlone M. A global/local third-order Hermitian displacement field with damaged interfaces and transverse extensibility: analytical formulation. Composite Structures 2003;59(4):419-31.

[29] Di Sciuva M, Gherlone M. A global/local third-order Hermitian displacement field with damaged interfaces and transverse extensibility: FEM formulation. Composite Structures 2003;59(4):433-44.

[30] Di Sciuva M, Gherlone M. Quasi-3D static and dynamic analysis of undamaged and damaged sandwich beams. Journal of Sandwich Structures \& Materials 2005;7(1):31-52. 
[31] Di Sciuva M, Gherlone M. Thermo-mechanics of undamaged and damaged multilayered composite plates: assessment of the FEM sub-laminates approach. Composite Structures 2007;81(1):137-55.

[32] Gherlone M, Di Sciuva M. Dynamic behaviour of undamaged and damaged multilayered composite plates. Key Engineering Materials 2007;347:531-6.

[33] Di Sciuva M, Gherlone M, Mattone M. Anisotropic cubic hermitian polynomialsand their use in the theory of laminated plates. Composite Structures. 2009; 88:304-311.

[34] Cesnik, C. E. S. and Hodges, D. H., "VABS: A new concept for composite rotor blade cross-sectional modeling," Journal of the American Helicopter Society, 1997; 42(1):27-38.

[35] Silvestre, N., and Camotim, D. (2002). "First-order generalized beam theory for arbitrary orthotropic materials.” Thin-Walled Structures, 40(9):791-820.

[36] Carrera E, Giunta G. Refined beam theories based on Carrera's unified formulation. International Journal of Applied Mechanics 2010; 2(1):117-43.

[37] Catapano A, Giunta G, Belouettar S, Carrera E. Static analysis of laminated beams via a unified formulation. Composite Structures 2011; 94(1):75-83.

[38] Pagano NJ. Exact solutions for composite laminates in cylindrical bending. Journal of Composite Materials 1969; 3:398-411. 


\section{Appendix}

The strain matrices $\mathbf{B}_{u}$ and $\mathbf{B}_{t}$ take the following explicit expression in term of the natural coordinate $\xi \in[-1,1]$

$$
\begin{gathered}
\mathbf{B}_{u}=\left[\begin{array}{llllllll}
-\frac{1}{l^{(e)}} & 0 & 0 & 0 & \frac{1}{l^{(e)}} & 0 & 0 & 0 \\
0 & \frac{6 \xi}{\left(l^{(e)}\right)^{2}} & \frac{(-1+3 \xi)}{l^{(e)}} & 0 & 0 & \frac{-6 \xi}{\left(l^{(e)}\right)^{2}} & \frac{(1+3 \xi)}{l^{(e)}} & 0 \\
0 & 0 & 0 & -\frac{1}{l^{(e)}} & 0 & 0 & 0 & \frac{1}{l^{(e)}}
\end{array}\right] \\
\mathbf{B}_{t}=\left[\begin{array}{llllllll}
0 & 0 & 0 & \frac{(1-\xi)}{2} & 0 & 0 & 0 & \frac{(1+\xi)}{2}
\end{array}\right]
\end{gathered}
$$

Integrals of Eq.(24) are developed as follows:

$$
\begin{gathered}
\iint_{A} E^{k} d A=\sum_{k=1}^{n_{\text {lauers }}} E^{k} b^{k} h^{k} \\
\iint_{A}-E^{k} z d A=-\sum_{k=1}^{n_{\text {lauers }}} \frac{E^{k} b^{k}}{2}\left(z_{k}^{2}-z_{k-1}^{2}\right) \\
\iint_{A} E^{k} z^{2} d A=\sum_{k=1}^{n_{\text {lauers }}} \frac{E^{k} b^{k}}{3}\left(z_{k}^{3}-z_{k-1}^{3}\right) \\
\iint_{A} E^{k} \phi^{k} d A=\iint_{A} E^{k}\left[-Z+\left(\bar{\phi}_{s}^{k-1}+\bar{\phi}_{d}^{k-1}\right)+\beta^{k}\left(z-z_{k-1}\right)\right] d A= \\
\sum_{k=1}^{n_{\text {lauers }}} E^{k} b^{k}\left[-\frac{1}{2}\left(z_{k}^{2}-z_{k-1}^{2}\right)+\left(\bar{\phi}_{s}^{k-1}+\bar{\phi}_{d}^{k-1}\right) h^{k}+\frac{\beta^{k}}{2}\left[\left(z_{k}^{2}-z_{k-1}^{2}\right)-z_{k-1} h^{k}\right]\right]
\end{gathered}
$$

where $\beta^{k}=\beta_{s}^{k}+\beta_{d}$ and $\bar{\phi}_{s}^{k-1}=\sum_{i=1}^{k-1} \beta_{s}^{i} h^{i}$

$$
\begin{gathered}
\iint_{A}-E^{k} z \phi^{k} d A=\iint_{A}-E^{k}\left[-z^{2}+\left(\bar{\phi}_{s}^{k-1}+\bar{\phi}_{d}^{k-1}\right) z+\beta^{k}\left(z^{2}-z_{k-1} z\right)\right] d A= \\
-\sum_{k=1}^{n_{\text {lauers }}} E^{k} b^{k}\left[-\frac{1}{3}\left(z_{k}^{3}-z_{k-1}^{3}\right)+\left(\bar{\phi}_{s}^{k-1}+\bar{\phi}_{d}^{k-1}\right) \frac{\left(z_{k}^{2}-z_{k-1}^{2}\right)}{2}+\beta^{k}\left[\frac{\left(z_{k}^{3}-z_{k-1}^{3}\right)}{3}-z_{k-1} \frac{\left(z_{k}^{2}-z_{k-1}^{2}\right)}{2}\right]\right] \\
\iint_{A} E^{k}\left(\phi^{k}\right)^{2} d A=\iint_{A} E^{k}\left[-z+\left(\bar{\phi}_{s}^{k-1}+\bar{\phi}_{d}^{k-1}\right)+\beta^{k}\left(z-z_{k-1}\right)\right]^{2} d A= \\
\sum_{k=1}^{n_{\text {lauers }}} E^{k} b^{k}\left[a_{1}^{k}\left(z_{k}^{3}-z_{k-1}^{3}\right)+a_{2}^{k}\left(z_{k}^{2}-z_{k-1}^{2}\right)+a_{3}^{k} h^{k}\right]
\end{gathered}
$$


with

$$
\begin{gathered}
a_{1}^{k}=\frac{\left(\beta^{k}-1\right)^{2}}{3} \\
a_{2}^{k}=\left(\beta^{k}-1\right)\left(\bar{\phi}_{s}^{k-1}+\bar{\phi}_{d}^{k-1}\right)-\left(\beta^{k}\right)^{2} z_{k-1}+\beta^{k} z_{k-1} \\
a_{3}^{k}=\left[\beta_{s}^{k} z_{k-1}-\left(\bar{\phi}_{s}^{k-1}+\bar{\phi}_{d}^{k-1}\right)\right]^{2}
\end{gathered}
$$

